\title{
Uncertainty Quantification and Sensitivity Analysis with CASL Core Simulator VERA-CS
}

\author{
C.S. Brown ${ }^{1}$, Hongbin Zhang ${ }^{2 *}$ \\ ${ }^{1}$ Department of Nuclear Engineering, North Carolina State University \\ Campus Box 7909, Raleigh, NC 27695-7909 \\ ${ }^{2}$ Idaho National Laboratory, P.O. Box 1625, Idaho Falls, ID 83415-3870, USA \\ csbrown3@ncsu.edu; Hongbin.Zhang@inl.gov
}

\begin{abstract}
VERA-CS (Virtual Environment for Reactor Applications, Core Simulator) is a coupled neutron transport and thermal-hydraulics code under development by the Consortium for Advanced Simulation of Light Water Reactors (CASL). An approach to uncertainty quantification and sensitivity analysis with VERACS was developed and a new toolkit was created to perform uncertainty quantification and sensitivity analysis. A 2x2 fuel assembly arrangement model was developed and simulated by VERA-CS, and uncertainty quantification and sensitivity analysis were performed with fourteen uncertain input parameters. The minimum departure from nucleate boiling ratio (MDNBR), maximum fuel center-line temperature, and maximum outer clad surface temperature were chosen as the selected figures of merit. Pearson, Spearman, and partial correlation coefficients were considered for all of the figures of merit in sensitivity analysis and coolant inlet temperature was consistently the most influential parameter. Parameters used as inputs to the critical heat flux calculation with the W-3 correlation were shown to be the most influential on the MDNBR, maximum fuel center-line temperature, and maximum outer clad surface temperature.
\end{abstract}

\section{INTRODUCTION}

Increased modeling fidelity of light water nuclear reactor behavior improves reactor safety and operational calculations. However, proper inputs to mathematical models of reactor neutronics and thermal-hydraulics are necessary to provide reliable and physical results. Uncertainty quantification (UQ) and sensitivity analysis (SA) methods seek to improve knowledge and understanding of a considered model for nuclear reactor behavior. Uncertainty quantification refers to the determination of uncertainty in model outputs based on the uncertainty in model inputs [1]. Sensitivity analysis seeks to determine the contribution of the uncertainty in a single model input to the uncertainty in model results [1]. Both UQ and SA are important in nuclear reactor calculations and seek to characterize the epistemic uncertainty associated with a mathematical model. Epistemic uncertainty results from the inability to know the correct value for a model input that is assumed constant [2].

Ikonen [3] compared a number of global sensitivity analysis methods by use of the nuclear fuel performance code VTT-modified FRAPCON-3.4. Global sensitivity analysis methods explore the whole input parameter space by sampling chosen input parameters simultaneously. In Monte Carlo based methods, a large number of model simulations are performed to produce a significant number of samples that can be used for both uncertainty quantification and sensitivity analysis. Marcum and Brigantic [2] performed a Monte Carlo uncertainty and global sensitivity analysis for the Multi-Application Small Light Water Reactor using the thermal-hydraulic codes RELAP5-3D and VIPRE-01. In both of these studies [2] [3], the reactor power characteristics (power shape, peaking factors, etc.) are inputs to the thermal-hydraulic codes. Marcum and Brigantic [2] found the axial and radial power factors to be the

\footnotetext{
${ }^{*}$ Corresponding author
} 
most influential parameters on the minimum departure from nucleate boiling ratio (MDNBR) and still highly influential on maximum fuel center-line and pin cladding temperature. Using constant inputs to the thermal-hydraulic code for core power characteristics does not allow neutronic/thermal-hydraulic coupling that could influence model results. Therefore, it is of interest to use fully-coupled neutronics and core thermal-hydraulic calculations to fully perform nuclear reactor core uncertainty quantification and sensitivity analysis. However, coupled calculations pose a challenge to Monte Carlo analysis since, in general, they require more computational power and longer simulation times than purely thermalhydraulic calculations.

In this work, the Virtual Environment for Reactor Applications, Core Simulator (VERA-CS) under development by the Consortium for Advanced Simulation of Light Water Reactors (CASL) is used to simulate a 2x2 nuclear fuel assembly arrangement model. VERA-CS (section 2) is a fully coupled core neutron transport and thermal-hydraulics code with the ability to model fuel depletion. An approach to uncertainty quantification and sensitivity analysis with VERA-CS is developed and applied to the 2x2 model.

\section{VERA-CS}

VERA-CS includes three main components: MPACT for reactor physics and neutron transport, COBRATF for thermal-hydraulics, and ORIGEN for isotopic depletion [4]. VERA-CS has been applied to an array of problems including core physics analysis [5], full-core modeling for the first cycle of Watts Bar Nuclear Unit 1 (WBN1) [6], and full-core modeling for all of the fuel cycles of WBN1 [7].

MPACT is a 3D pin-resolved reactor transport code developed by Oak Ridge National Laboratory (ORNL) and the University of Michigan. MPACT uses the 2D/1D method to solve the neutron flux distribution throughout the specified geometry. In the 2D/1D method, the 2D method of characteristics (MOC) is used in the radial planes in order to capture the heterogeneity in the radial direction with high accuracy; where each pin cell is explicitly modeled and even sub-pin detail can be captured [4]. In the axial direction a low-order transport solution using $\mathrm{SP}_{3}$ is obtained through a pin-cell homogenized basis [4]. Axial and radial solutions are linked through the use of transverse leakage terms that ensure neutron balance in every pin-cell at convergence [4]. MPACT uses a 47 energy group library based on ENDF/B VII data with subgroup parameters to capture self-shielding effects [4]. More details on MPACT methodology can be found in references [4], [6], and [8].

COBRA-TF (CTF) is a 9-equation subchannel analysis code developed by ORNL and Pennsylvania State University [9]. CTF solves the mass, momentum, and energy equations for the liquid, vapor, and droplet phases for each subchannel in the core and captures the axial flow within each channel as well as crossflow between channels. Direct coupling between neutronics (MPACT) and thermal-hydraulics (CTF) in VERA-CS provides temperature and density feedback. CTF includes a number of models important for LWR safety and design analysis including flow regime dependent two-phase wall heat transfer, interphase heat transfer and drag, droplet breakup, and quench-front tracking [6]. A more in-depth discussion on the coupling between MPACT and CTF can be found in reference [6].

ORIGEN [10] is developed by ORNL and released through the SCALE package. ORIGEN is directly integrated in MPACT through its API to calculate the changes in nuclides for every depletable crosssection region [4].

\section{PROBLEM DESCRIPTION}

Nuclear reactor licensing has long focused on the main steam line break (MSLB) accident. Recently, work within CASL [11] used VERA-CS to assess the core response to a MSLB event that poses a 
challenge to the departure from nucleate boiling (DNB) criteria. The postulated accident was initiated from low operating power condition at the end of cycle 1 for a typical PWR nuclear power plant. In the accident scenario, increased steam flow rate from the broken steam pipe on one of the steam generators results in a significant reduction in the primary coolant temperature and an increase in the reactor core average power and peak fuel rod power [11]. The analysis results from a system analysis code for the MSLB scenario were used as the boundary conditions to carry out the high fidelity reactor core response simulations using VERA-CS.

The computational resources required to perform full-core simulations with VERA-CS are not affordable for a Monte Carlo based sensitivity analysis. Therefore, the current work focuses on a 2x2 nuclear fuel assembly arrangement model (Figure 1) with parameters defined by a full-core simulation and adjusted for the smaller geometry. Table 1 shows the core design parameters for a typical pressurized water reactor (PWR) during hot full power operation. Fuel designs are typical 17x17 fuel assemblies with 264 fuel rods, 24 guide tubes, and 1 instrumentation tube.

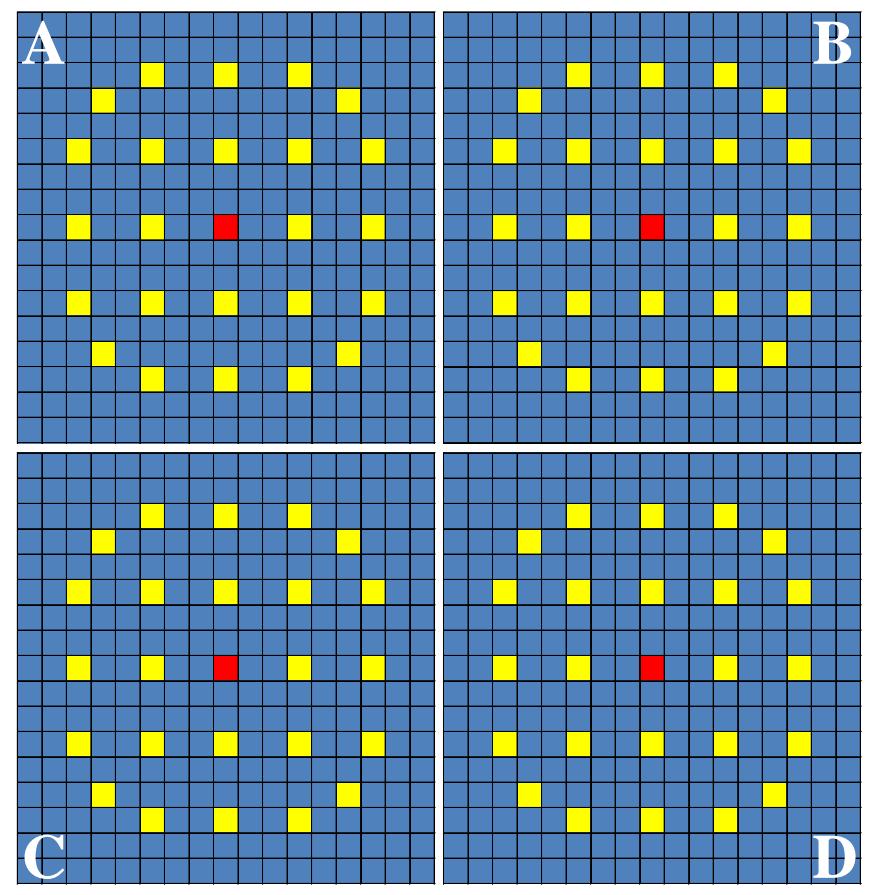

Figure 1 . The 2x2 model with each assembly labeled (A-D). The yellow cells represent locations of guide tubes, the red cells indicate locations of instrumentation tubes (center of assembly), and blue cells represent fuel pin locations.

Table 1. Typical PWR core design parameters at hot full power conditions.

\begin{tabular}{|c|c|c|}
\hline Parameter & Value & Unit \\
\hline Core Power & 3411 & $\mathrm{MW}_{\text {th }}$ \\
\hline Inlet Temperature & 544 & ${ }^{\circ} \mathrm{F}$ \\
\hline Inlet Flow Rate & 178.054 & $\mathrm{lb} / \mathrm{s}$ \\
\hline System Pressure & 2250 & $\mathrm{psia}$ \\
\hline
\end{tabular}

The 2x2 model was setup using the $17 \mathrm{x} 17$ fuel assemblies with all assembly parameters (Table 2) obtained from open literature. VERA-CS takes into account core and fuel assembly properties such as the upper and lower core plates, lower and upper assembly nozzles, and guide/instrument tubes. Eight spacer 
grids are included in the assemblies to provide structural support as well as improve coolant mixing. The spacer grid form losses are a user input to the VERA-CS input file.

The 2x2 model also used the VERA-CS capability to model control rod effects by placing control rods in each of the four assemblies. However, it is assumed that the control rod bank in the top right assembly (assembly $B$ ) is stuck completely out of the core to facilitate an increase in core power and test the safety limits for this particular geometry. Reflective boundary conditions were used in the radial direction.

Table 2. Typical 17x17 PWR fuel assembly parameters used in VERA-CS.

\begin{tabular}{|c|c|c|}
\hline Parameter & Value & Unit \\
\hline Active Core Length & 365.76 & $\mathrm{~cm}$ \\
\hline Rod Pitch & 1.26 & $\mathrm{~cm}$ \\
\hline Clad Material & ZIRC & $\mathrm{cm}$ \\
\hline Clad Outer Radius & 0.475 & $\mathrm{~cm}$ \\
\hline Clad Inner Radius & 0.418 & - \\
\hline Fuel Material & $\mathrm{UO}_{2}$ & $\mathrm{~cm}$ \\
\hline Fuel Pellet Outer Radius & 0.4096 & - \\
\hline Instrument Tube Material & ZIRC & $\mathrm{cm}$ \\
\hline Instrument Tube Thickness & 0.041 & $\mathrm{~cm}$ \\
\hline Instrument Tube Outer Radius & 0.602 & $\mathrm{~cm}$ \\
\hline Assembly Pitch & 21.5 & \\
\hline
\end{tabular}

Uniform inlet temperature and mass flux distributions were used for this geometry although VERA-CS has the capability to include inlet temperature and mass flux distributions on a per-assembly basis. The $2 \times 2$ model in the current work is a rough representation of the area of the stuck rod region in the full-core simulation performed in reference [11]. However, future work will focus on better representation of the stuck rod region since this work aims to develop an uncertainty quantification and sensitivity analysis procedure for use with VERA-CS.

The VERA-CS input [12] provides the user with options for controlling parameters within CTF. The chf flag was set equal to one for the $2 \times 2$ case so that the $\mathrm{W}-3$ boiling correlation [13] was used to calculate the critical heat flux.

CTF uses a turbulent mixing coefficient $\left(\beta_{\mathrm{sp}}\right)$ that can either be set to some constant value or defined by the CTF equations [14]. This turbulent mixing coefficient is a multiplier of the calculated transverse velocity and a nominal value of 0.005 was used in this work. The void drift cross-flow is calculated in a similar manner to turbulent mixing cross-flow [14], where again there is a multiplicative constant that can be controlled by the user with the VERA-CS input file. A nominal value of 1.4 for the void drift model coefficient $\left(K_{m}\right)$ was used in this work. Note that setting $K_{m}$ equal to zero would result in no void drift modeling within CTF.

The VERA-CS input file also provides the user with an option to define the amount of power deposited directly in the coolant, or gamma heating, as the dhfrac input parameter. A nominal value of 0.02 for the dhfrac parameter was used in this work. 


\section{METHODOLOGY}

\subsection{Figures of Merit}

Performing uncertainty and sensitivity analysis requires that a relevant system response output variable, or figure of merit (FOM), be chosen to analyze. VERA-CS provides a number of system response variables that could be analyzed. In this work, three outputs that pertain to U.S. NRC licensing regulations are chosen as figures of merit: the MDNBR, the maximum fuel center-line temperature, and the maximum outer clad surface temperature. The maximum fuel center-line and outer clad surface temperatures are quantities directly obtained from the VERA-CS solution. However, the MDNBR is the minimum departure from nucleate boiling ratio (DNBR, Eq. 1) calculated anywhere within the reactor core. DNBR is a derived value based on the calculated critical heat flux at a specific location.

A departure from nucleate boiling (DNB) event occurs in a reactor subchannel when the local heat flux through the heated wall to the coolant reaches the critical heat flux value. In a DNB scenario, the inability for the coolant to reach the wall surface due to increased vapor formation can result in overheating of the clad and fuel rod to the point of failure. Therefore, it is of interest in reactor performance analysis codes to calculate the minimum DNBR (MDNBR) everywhere within the reactor core as a safety metric and insure that this value remains above a predetermined safety standard.

$$
D N B R=\frac{q_{C H F}^{\prime \prime}}{q_{\text {local }}^{\prime \prime}}
$$

When the $\operatorname{chf}$ flag is set equal to one in the VERA-CS input file CTF calculates the critical heat flux value at each node using the W-3 correlation. The W-3 correlation was developed by Tong et al. at the Westinghouse Electric Company [13] and calculates the critical heat flux as a function of inlet subcooling, pressure, and coolant mass flux. When the VERA-CS input file flag edit_dnb is set equal to one a summary file of the heat flux, critical heat flux, and DNBR at each node is output for each assembly.

\subsection{Input Uncertainty}

Marcum and Brigantic (2015) note that the International Atomic Energy Agency (IAEA) has identified three major sources of uncertainty in thermal hydraulic calculations: code or model uncertainty, representation of simulation uncertainty, and plant uncertainty. The focus of this work is on plant uncertainty as the uncertainty in user inputted values to the VERA-CS input file, or the epistemic uncertainty [1].

The specific number of input parameters to consider in uncertainty quantification is sometimes defined by the analysis method itself. In non-parametric methods, such as the Monte Carlo approach used in the current work, the amount of parameters is not specified by the method itself but there is still motivation to limit the number of uncertain parameters [2]. The specific parameters should be judiciously chosen based on those that are expected to have the greatest epistemic uncertainty or the most influence on the FOMs for sensitivity analysis. A table of uncertain parameters can be developed that indicates the most important inputs to the model and their expected range of uncertainty (Table 3). Correlation between model inputs will have an effect on how these parameters are sampled for uncertainty quantification and sensitivity analysis.

The uncertain parameters (Table 3) for UQ/SA with VERA-CS have been chosen as those that are expected to influence the figures of merit or have substantial input uncertainty. It is intuitive to include 
the inlet coolant temperature, system pressure, and inlet coolant mass flux as sources of uncertainty since the W-3 correlation is a function of these variables. Similar values for these parameters were used in references [2] and [3]. In general, studies of reactor response [2] [15] [16] must include core power parameters (e.g. power distribution and peaking factors) as inputs to the thermal-hydraulic code as sources of uncertainty. However, the fully-coupled neutronic and thermal-hydraulic capabilities of VERA-CS eliminate this major source of input uncertainty since the core power distribution is calculated directly and thermal-hydraulic feedback is considered. This is an important step in eliminating uncertainty in reactor safety and operation calculations since the effect of perturbed variables on the power shape is captured by direct calculation. The reactor operating power is an important parameter that has some uncertainty range in MSLB scenarios and plays a major role in the calculation of the linear heat rate and rod heat flux and is therefore expected to influence the FOMs.

Table 3. Table of uncertain parameters.

\begin{tabular}{|c|c|c|c|}
\hline Uncertain Parameter & Nominal Value & $\begin{array}{l}\text { Uncertain } \\
\text { Range }\end{array}$ & Distribution \\
\hline Core Power & $25.3 \%$ of rated & $\pm 2 \%$ & Uniform \\
\hline Inlet Coolant Temperature & $429.4^{\circ} \mathrm{F}$ & $\pm 2^{\circ} \mathrm{F}$ & Uniform \\
\hline System Pressure & 518.49 psia & $\pm 1 \%$ & Uniform \\
\hline Inlet Coolant Mass Flow Rate & $\begin{array}{c}\text { 164.49 Mlbs/hr } \\
\text { (3.410 Mlbs/hr when rated } \\
\text { for } 4 \text { assemblies) }\end{array}$ & $\pm 3 \%$ & Uniform \\
\hline $\begin{array}{l}K_{m} \text { : distribution weighting factor } \\
\text { for the CTF void-drift model }\end{array}$ & ( & $\pm 10 \%$ & Uniform \\
\hline $\begin{array}{c}\beta_{\mathrm{sp}}: \text { CTF constant turbulent } \\
\text { mixing coefficient }\end{array}$ & 0.005 & $\pm 10 \%$ & Uniform \\
\hline $\begin{array}{l}\text { dhfrac: Fraction of power } \\
\text { deposited directly in coolant }\end{array}$ & 0.02 & $\pm 10 \%$ & Uniform \\
\hline Clad outer/inner radius & $0.475 / 0.418$ & $\pm 0.002 \mathrm{~cm}$ & Uniform \\
\hline Fuel pellet radius & 0.4096 & $\pm 0.001 \mathrm{~cm}$ & Uniform \\
\hline Fuel \% of theoretical density & $94.5 \%$ & $\pm 1.6 \%$ & Uniform \\
\hline Fuel enrichment (\%) & $3.10 \%$ & $\pm 0.003 \%$ & Uniform \\
\hline $\begin{array}{c}\text { Spacer grid form loss - } \\
\text { END/MID }\end{array}$ & $0.9070 / 0.9065$ & $\pm 20 \%$ & Uniform \\
\hline
\end{tabular}

The void-drift model coefficient $\left(K_{m}\right)$, turbulent mixing coefficient $\left(\beta_{\mathrm{sp}}\right)$, and spacer grid form losses are all based on best-estimate models and included as source/sink terms in the CTF momentum equations. Therefore, each of these terms is prescribed a large uncertainty range. For the spacer grid form losses, END denotes those spacer grids at the top and bottom of the assembly and MID refers to the remaining six spacer grids spaced throughout the internal portion of the assembly. The spacer grid form loss inputs are correlated such that the same perturbation percentage is applied to both the END and MID distinctions. The same uncertainty range for the spacer grid form losses were used in previous nuclear thermal-hydraulic UQ/SA studies by Marcum and Brigantic [2]. Much like the models used for source/sink terms in the CTF momentum equations, the dhfrac term represents a source/sink term in the CTF energy equations and a sizable uncertainty range is applied to this input as well. The uncertain parameters pertaining to the fuel and cladding specifications were chosen based on fuel manufacturing and operational characteristics. In this VERA-CS case, fresh fuel is assumed and manufacturing tolerances are then applied to fuel and cladding specifications (identical or similar tolerances were used in reference [3]). For the inner and outer clad radii the inputs are correlated such that the same perturbation of the manufacturing tolerance is applied to both. Fuel density and enrichment uncertainties specified here are those from previous sensitivity analysis studies of fuel performance by Ikonen [3]. 
The uncertain parameters designated in Table 3 are sampled from a uniform distribution within the defined uncertain range. The uniform distribution is considered conservative since maximum and minimum values are equally as likely to occur as the nominal value [2]. Nominal values are chosen based on previous studies of MSLB where reactor pumps continue to operate with available offsite power. In this case, the inlet mass flow rate remains quite high, at around $20 \%$ of the full-operating value. Sampling from the uniform distribution is performed using the random.uniform method in Python. Section 4.4 outlines the overall analysis method for performing UQ/SA.

This work performed a Monte Carlo based approach to uncertainty quantification and a large number of samples were performed. Therefore, probability distribution functions (PDF) and cumulative distribution functions (CDF) can be computed for each of the FOMs. The PDF can indicate how the correlation between model inputs and model outputs behaves [3] while the CDF can be used to quantify the uncertainty in the FOMs [2].

\subsection{Sensitivity Analysis}

Sensitivity analysis seeks to determine the contribution of the uncertainty in specific inputs to the uncertainty in analysis results [1]. Results from sensitivity analysis provide a clearer picture of how system inputs correlate to system outputs. Parameters with negligible or no contribution to the system response can be removed in future studies while those parameters with significant contribution present a guide to where areas of future research should be focused on reducing the input uncertainty. Ikonen [3] has examined a number of global sensitivity analysis methods in application to nuclear fuel performance modeling. Global sensitivity analysis explores the whole input parameter space [3] rather than performing perturbations of input parameters one-at-a-time (OAT) such as in the OAT method. There exist numerous sensitivity analysis methods [1] [3] that should be carefully chosen based on the complexity and specific model to be evaluated. In this work, a Monte Carlo, or sampling based, approach is used to evaluate those parameters that most profoundly affect the FOMs.

Scatterplots are often the first step to examine the relationship between the uncertainty in model inputs and analysis results while revealing any non-linearity or unexpected behavior [1]. Scatterplots provide the starting point for development of a more qualitative sensitivity analysis strategy. Rank transformed data can also be used to create scatterplots when the data exhibits a non-linear yet monotonic relationship [2]. Rank transformation is used to rank the input and output data from the smallest values, with a rank of 1 , to the largest values with a rank corresponding to the number of samples. Rank transformed scatterplots are then formed by plotting the rank transformed output data $y$ versus the rank transformed input data $x$.

Although scatterplots are instrumental in examining the relationship between the model input and output parameters, qualitative methods such as correlation coefficients provide the degree of linearity that exists between inputs and outputs. Various methods for computing correlation coefficients exist in the literature [1] [3] and the method used must be sensibly chosen based on the sensitivity analysis approach. Correlation coefficients are valued between -1 and +1 where -1 represents a perfect inversely correlated linear relationship and +1 represents a perfect linear relationship. A value close to 0 indicates that the input has insignificant effect on the output. Absolute values of the correlation coefficients between model inputs and a particular FOM can then be ranked from those inputs that are the most influential to those

that are the least influential on the FOM. The Pearson (or sample) correlation coefficient (CC) between inputs $x_{j}$ and output $y$ as defined by Helton et al. [1] is:

$$
c\left(x_{j}, y\right)=\frac{\sum_{i=1}^{N}\left(x_{i j}-\bar{x}_{j}\right)\left(y_{i}-\bar{y}\right)}{\left[\sum_{i=1}^{N}\left(x_{i j}-\bar{x}_{j}\right)^{2}\right]^{1 / 2}\left[\sum_{i=1}^{N}\left(y_{i}-\bar{y}\right)^{2}\right]^{1 / 2}}
$$


where

$$
\begin{gathered}
\bar{x}_{j}=\sum_{i=1}^{N} \frac{x_{i j}}{N} \\
\bar{y}=\sum_{i=1}^{N} \frac{y_{i}}{N}
\end{gathered}
$$

and $N$ is the number of samples. The Pearson correlation coefficient can also be applied to the rank transformed data and is then known as the Spearman, or rank, correlation coefficient (RCC).

In a global sensitivity analysis approach, perturbations in the model output are not purely a function of that of a single input, but rather a combinational effect from the perturbation of all model inputs simultaneously. To evaluate the comprehensive quality of the sensitivity analysis the square of the Pearson correlation coefficient $\left(R^{2}\right)$ can be calculated for each input parameter and summed. If the value remains significantly below unity then higher order sensitivity analysis methods, such as partial correlation coefficients (PCCs), must be used to analyze nonlinearities in the model [3]. PCCs characterize the linear relationship between a model input and model output after corrections have been made for the linear effects on the output by all other model inputs.

\subsection{VERA-CS Uncertainty and Sensitivity Analysis Toolkit}

The overall computational method to perform the uncertainty and sensitivity analysis (Figure 2) forms the VERA-CS Uncertainty and Sensitivity Analysis Toolkit (VUSAT). VUSAT contains two main portions.

In Figure 2 the solid lines indicate the first VUSAT portion of pre-processing and VERA-CS execution. This pre-processing step uses a Python script to perform the overall perturbation of the nominal values. A VERA-CS input file was first created for the 2x2 case with the nominal input values shown in Table 3. The table of uncertain parameters was also created as an input to the Python script. The Python script is then called to read the nominal VERA-CS input file, sample the variables defined in the table of uncertain parameters from the ranges defined in the table of uncertain parameters input file, and then create $N$ new VERA-CS input files with perturbed parameters. Each of these input files is placed into a new directory in the Linux file system so that VERA-CS code output for each case is kept separate. A shell script was then used to submit all of the new perturbed cases to the Idaho National Laboratory (INL) high performance computing queue.

For each of the $N$ simulations a number of CTF output files are created for each of the four assemblies. Namely, a summary file of DNB parameters (i.e. heat flux, CHF, DNBR), an output file with results (i.e. equilibrium quality, mass flux, void fraction, etc.) for each channel within the assembly, and an output file of temperature information for each rod within the assembly. The post-processing portion of the VUSAT (dashed lines in Figure 2) utilizes both Python scripting and Fortran 95 coding to first search the DNB output files and find the MDNBR value and location. The VUSAT then searches the rod output files to find the maximum fuel center-line temperature and maximum outer clad surface temperature. Data from the channel where the MDNBR occurs is also written to an output file. The final step of the VUSAT retrieves the FOMs from each of the case summary files as well as the values for each of the perturbed parameters defined by the table of uncertain parameters input file. A FOM summary file is then written that contains the FOMs and perturbed values for each case. 


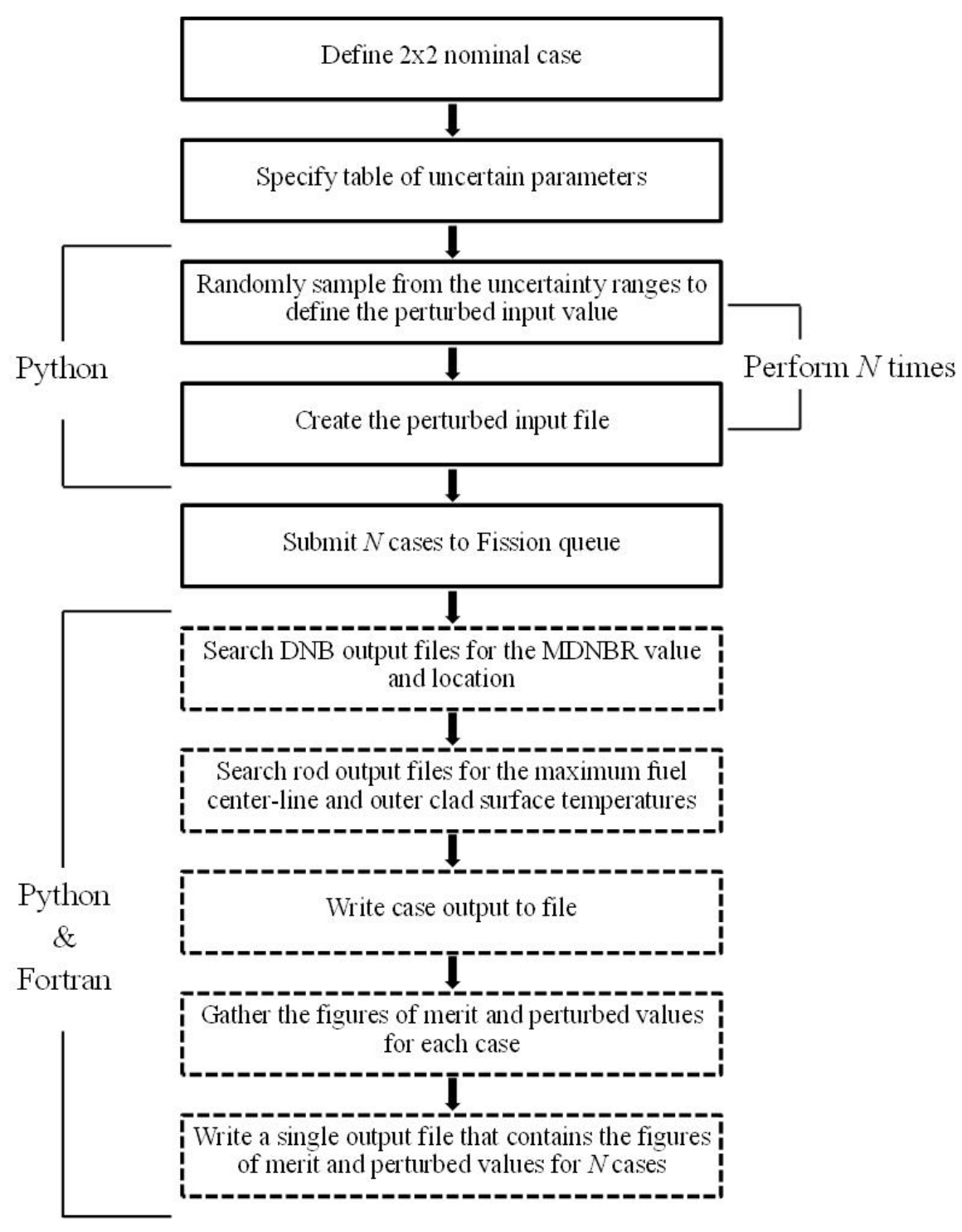

Figure 2. VUSAT summary. Solid lines indicate pre-processing and code execution steps while dashed lines indicate post-processing steps.

\section{$5 \quad$ RESULTS}

The VERA-CS 2x2 fuel assembly arrangement was developed for uncertainty quantification and sensitivity analysis. The UQ and SA simulations were performed on the Fission supercomputer at the INL. Fission is a 12,512 core Appro distributed memory system with 25 TB of total memory. A limited number of cases were performed using 232 cores. However, to take advantage of the ability of more cases to run in parallel with only a slight increase in time penalty, the majority of cases were performed on 58 cores with an average run time of 3.20 hours. The VUSAT described in section 4.4 was used to create 5200 VERA-CS input files with perturbed parameters outlined by the table of uncertain parameters (Table 3). Of these 5200 simulations, 5143 converged and are considered here. The VUSAT post-processor was then used to extract the FOMs, perturbed values, and any other quantities of interest. The nominal 2x2 case and the two cases with the maximum and minimum MDNBR values are first discussed. 


\subsection{VERA-CS Model Results}

VERA-CS results for the nominal case are shown here in comparison to results for the most and least restrictive cases based on the MDNBR value. Table 4 shows the values for the MDNBR and the fuel center-line and outer clad surface temperatures at the MDNBR location, as well as the perturbed parameters for each of these cases.

Table 4. Comparison of the FOMs and perturbed parameter values for the minimum MDNBR, nominal, and maximum MDNBR cases.

\begin{tabular}{|c|c|c|c|c|}
\hline & Parameter & $\begin{array}{c}\text { Most } \\
\text { Restrictive }\end{array}$ & Nominal & $\begin{array}{c}\text { Least } \\
\text { Restrictive }\end{array}$ \\
\hline \multirow{3}{*}{ لَّ } & MDNBR & 3.8015 & 4.6978 & 5.2502 \\
\hline & $\begin{array}{c}\text { Fuel Center-Line Temperature } \\
{\left[{ }^{\circ} \mathrm{F}\right] \text { (at MDNBR location) }}\end{array}$ & 2656.56 & 2259.08 & 2137.27 \\
\hline & $\begin{array}{c}\text { Outer Clad Surface Temperature } \\
{\left[{ }^{\circ} \mathrm{F}\right] \text { (at MDNBR location) }}\end{array}$ & 491.70 & 486.53 & 483.65 \\
\hline \multirow{14}{*}{ 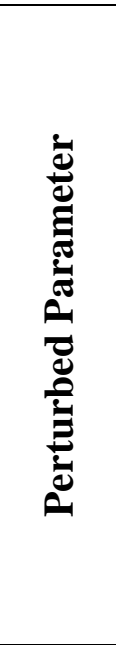 } & Power [\% of rated] & 25.7858 & 25.3 & 24.8601 \\
\hline & Inlet Temperature $\left[{ }^{\circ} \mathrm{F}\right]$ & 431.0319 & 429.4 & 427.6475 \\
\hline & System Pressure [psia] & 514.5983 & 518.49 & 520.4547 \\
\hline & Inlet Mass Flow Rate [Mlbs/hr] & 3.3115 & 3.410 & 3.4690 \\
\hline & $K_{m}$ & 1.4249 & 1.4 & 1.5247 \\
\hline & $\beta_{\mathrm{sp}}$ & 4.9332E-03 & $5.0 \mathrm{E}-03$ & 5.0454E-03 \\
\hline & dhfrac & $2.0262 \mathrm{E}-02$ & $2.0 \mathrm{E}-02$ & $2.0274 \mathrm{E}-02$ \\
\hline & Spacer Grid Form Loss - MID & 1.0868 & 0.9070 & 0.7376 \\
\hline & Spacer Grid Form Loss - END & 1.0862 & 0.9065 & 0.7372 \\
\hline & Fuel Pellet Radius [cm] & 0.410561 & 0.4096 & 0.409755 \\
\hline & Clad Inner Radius [cm] & 0.417385 & 0.4180 & 0.418323 \\
\hline & Clad Outer Radius [cm] & 0.473492 & 0.4750 & 0.476977 \\
\hline & Fuel \% of Theoretical Density & 95.46979 & 94.5 & 93.22656 \\
\hline & Fuel Enrichment [\%] & 3.100027 & 3.1 & 3.100054 \\
\hline
\end{tabular}

Figure 3 shows the DNBR as a function of the distance from the channel inlet for the location where the MDNBR occurred for each case. The bump in the plot around $0.6 \mathrm{~m}$ is due to effects from the first spacer grid. Interestingly, the bump 'height' corresponds with the value for the spacer grid form loss in each case, i.e. the bump is higher for the least restrictive case where spacer grid form losses were the smallest. The plot inset shows that although the most restrictive case experienced the lowest MDNBR, the DNBR was greater as axial distance from the inlet increased. This behavior is reflected in the fuel center-line (Figure 4) and outer clad surface (Figure 5) temperatures as well, where the most restrictive case experiences lower temperatures further from the channel inlet. 


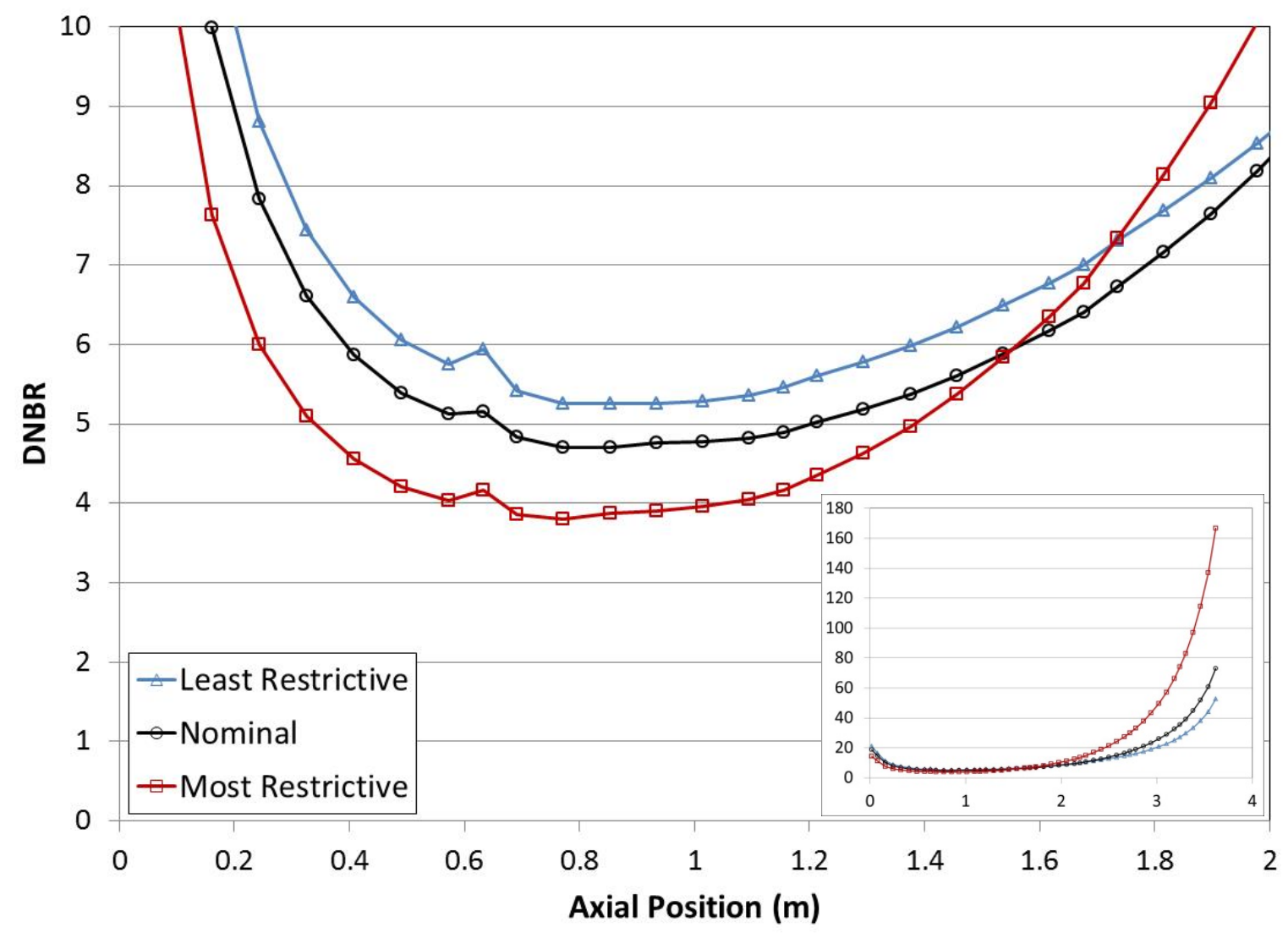

Figure 3. Zoomed DNBR for the first two meters of the channel as a function of distance from the channel inlet for the least restrictive (blue triangles), nominal (black circles), and most restrictive (red squares) cases. Shown for the channel where MDNBR was located. Plot inset shows DNBR over the full channel height.

VERA-CS results for total mass flow rate (Figure 6) and equilibrium quality (Figure 7) show expected trends for the least restrictive, nominal, and most restrictive cases. In Figure 6, the sharp decreases in total mass flow rate are due to the spacer grid effects in the channel. The difference in spacer grid form loss between the cases is evident since decreases in the most restrictive case are much more pronounced. In Figure 7, the most restrictive case experiences the least amount of subcooling (greatest equilibrium quality) which attributes to the lower MDNBR.

A comparison of the input values in Table 4 shows that each case behaves as expected. The most restrictive case happens where the most extreme of sampled values contribute to lowering the MDNBR. For example, the power, inlet temperature, and spacer grid form losses are all greater for this case in comparison to the nominal and least restrictive while the pressure, inlet mass flow rate, and clad outer radius are all less for the most restrictive case. This combination exacerbates the critical heat flux. The values of other parameters such as gamma heating and fuel enrichment are less for the most restrictive case than for the least restrictive case. However, results from sensitivity analysis (section 4.3) will show that these inputs are less influential on the MDNBR. 


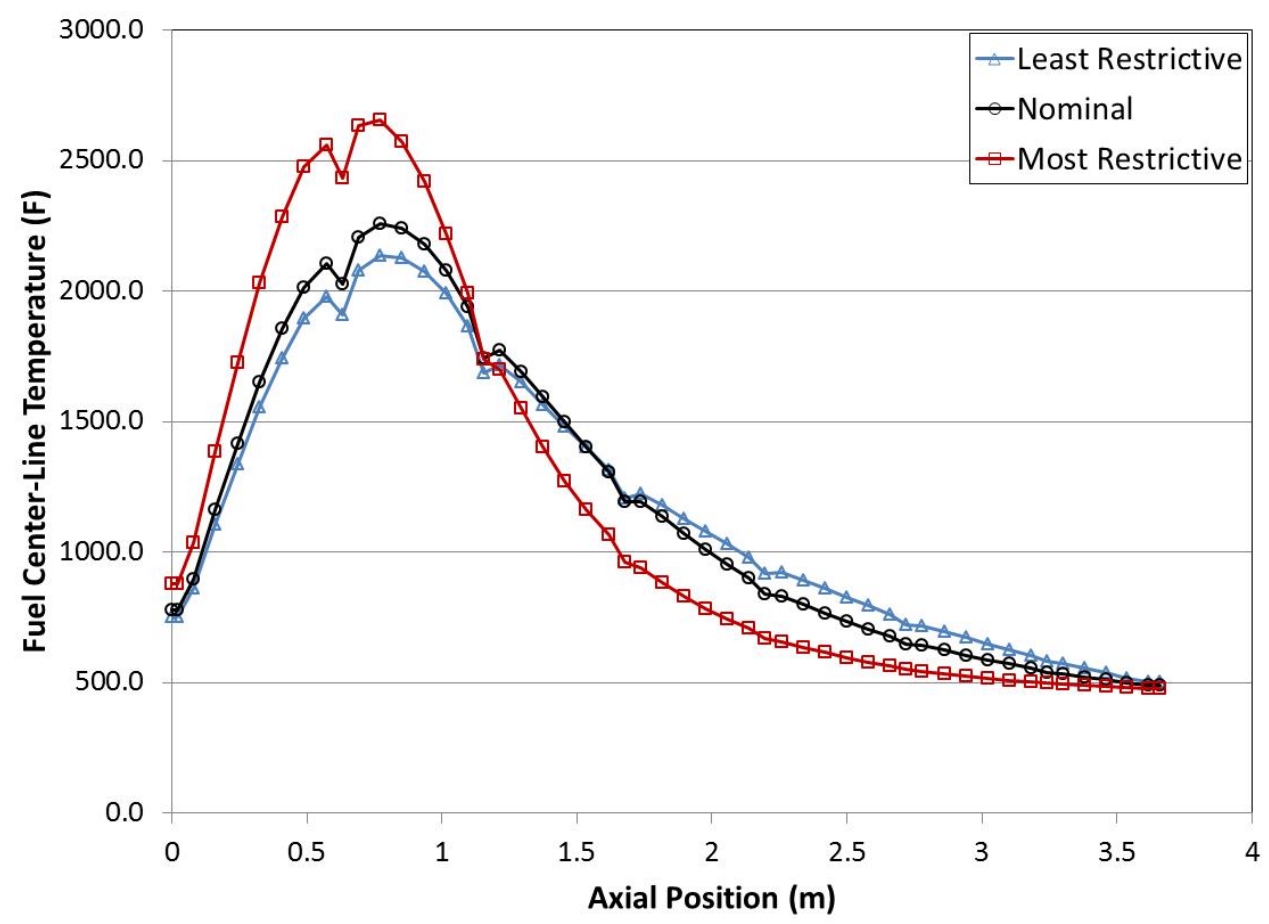

Figure 4. Fuel center-line temperature as a function of axial distance from the channel inlet for the least restrictive (blue triangles), nominal (black circles), and most restrictive (red squares) cases.

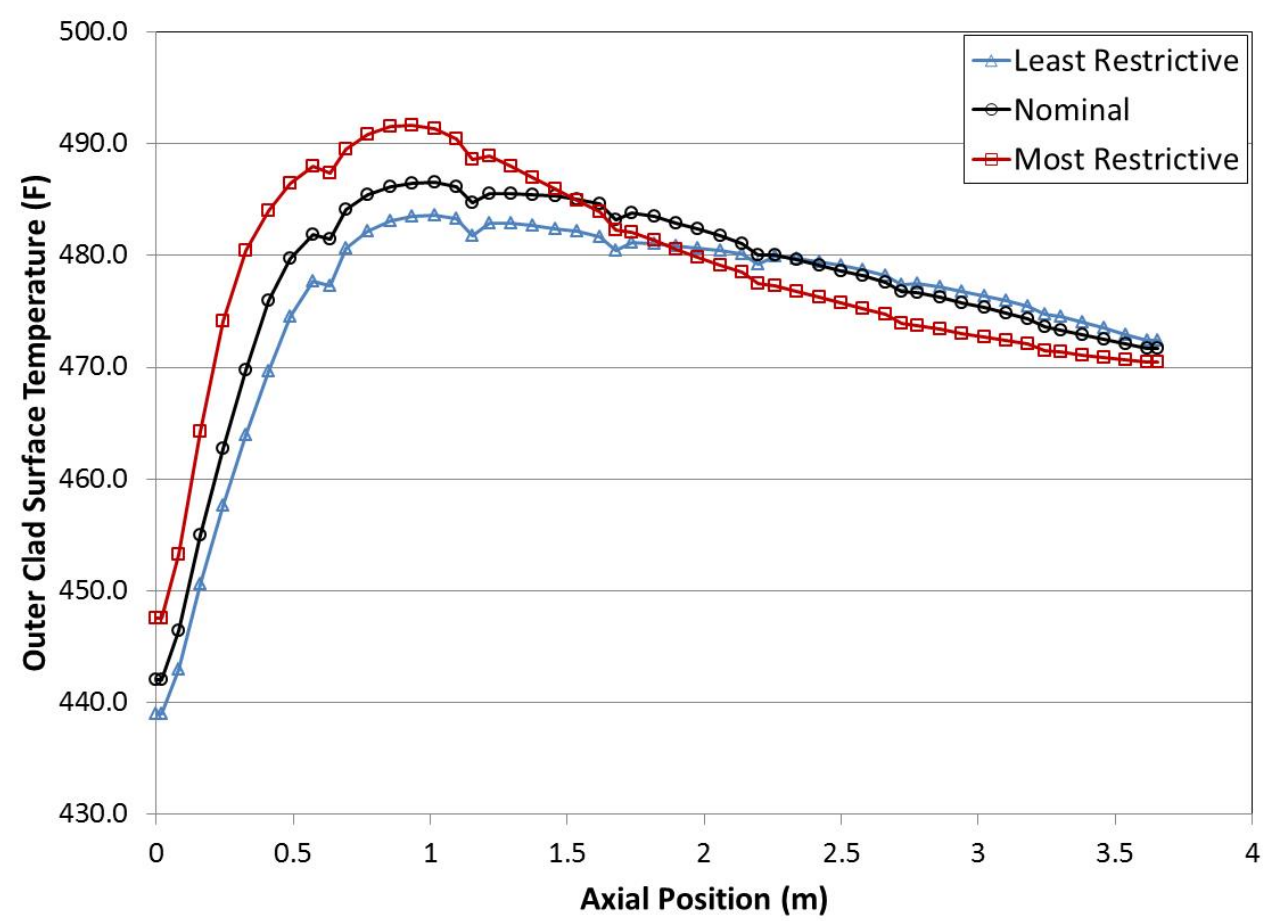

Figure 5. Outer clad surface temperature as a function of axial distance from the channel inlet for the least restrictive (blue triangles), nominal (black circles), and most restrictive (red squares) cases. 


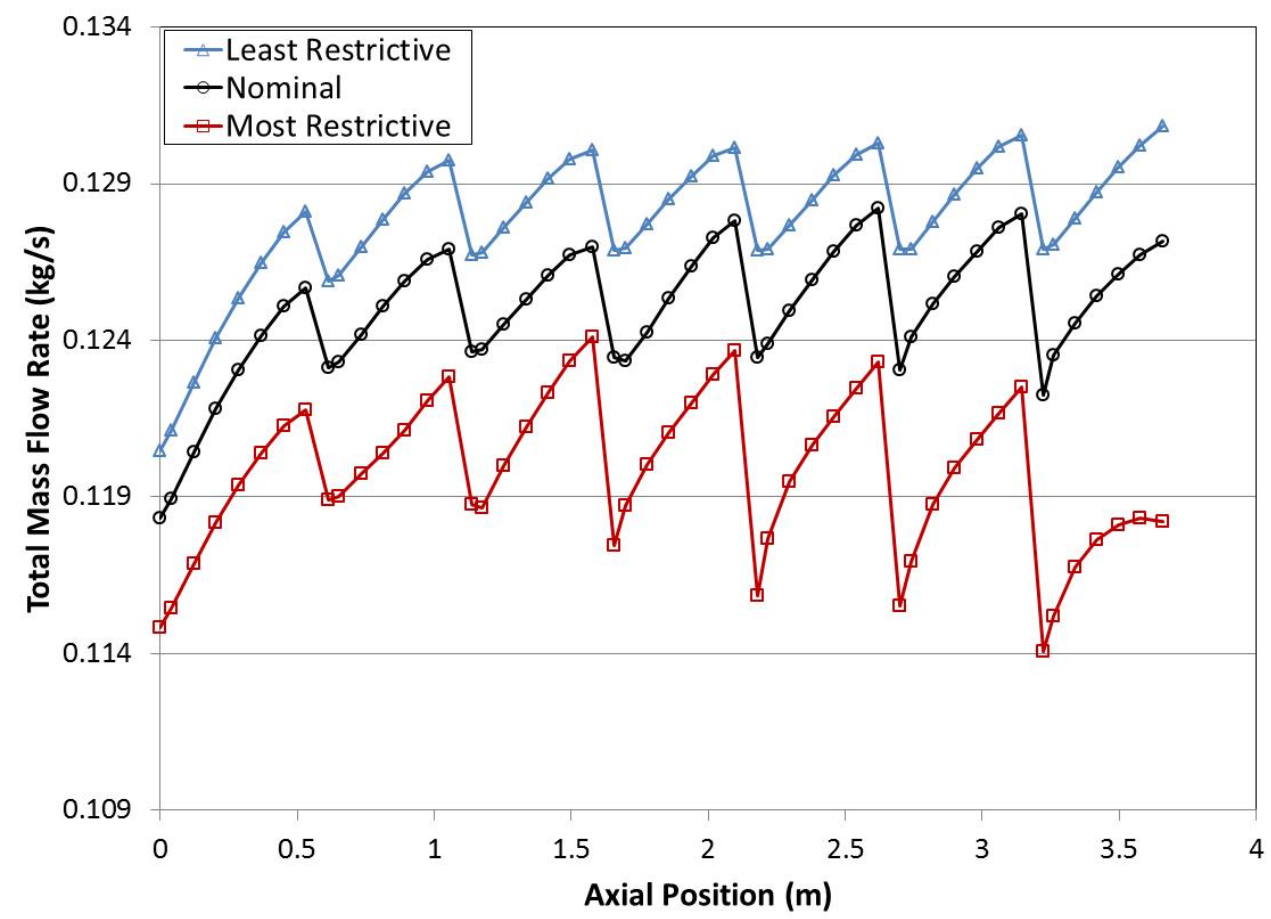

Figure 6. Total mass flow rate as a function of axial distance from the channel inlet for the least restrictive (blue triangles), nominal (black circles), and most restrictive (red squares) cases.

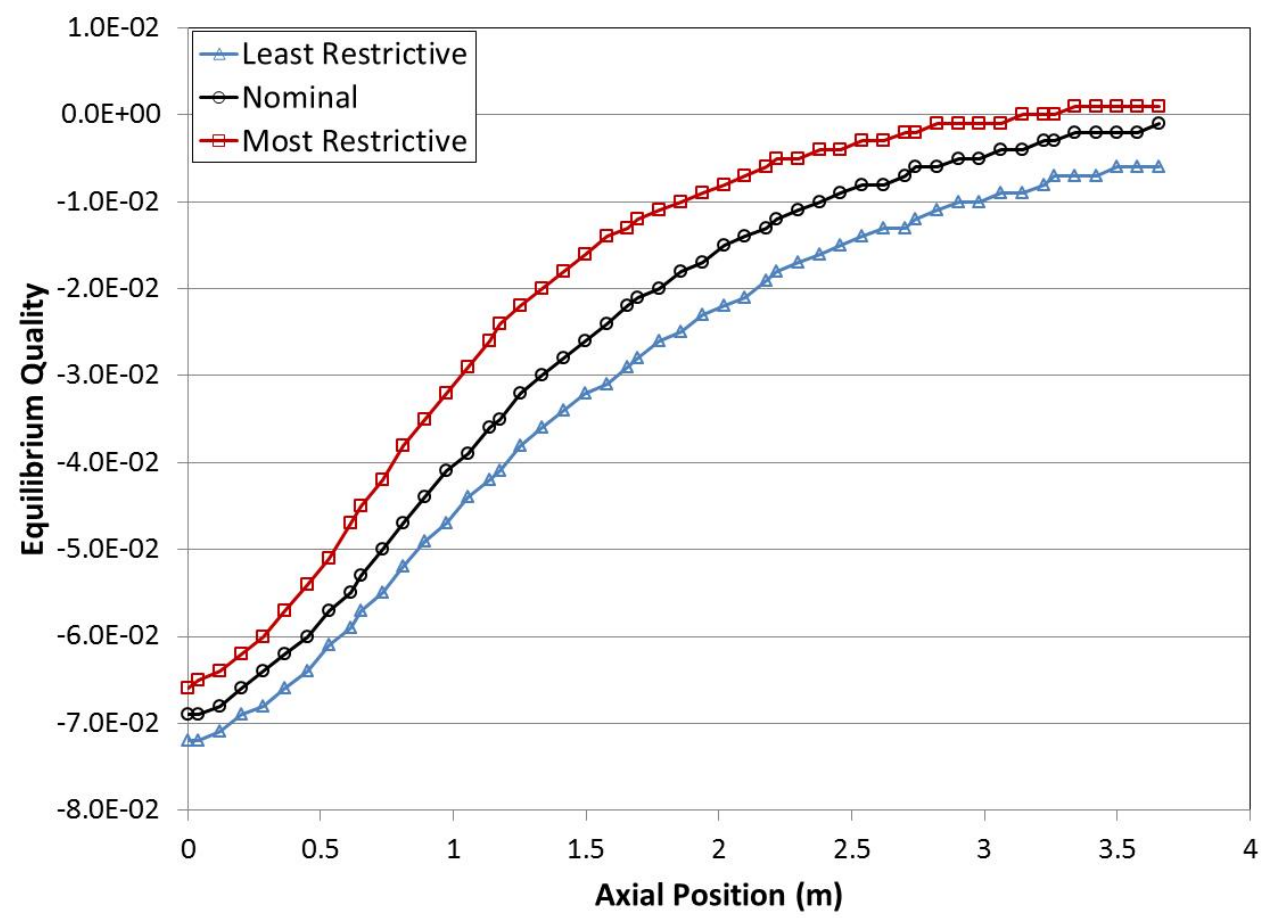

Figure 7. Equilibrium quality as a function of axial distance from the channel inlet for the least restrictive (blue triangles), nominal (black circles), and most restrictive (red squares) cases.

Figure 8 shows the pin power distribution for the most restrictive (a), least restrictive (b), and nominal (c) cases as well as the moderator temperature in the $2 \times 2$ model for the most restrictive case (d). The highest 
powered location in the model occurs in the upper right hand corner of assembly $B$ due to the combined effect of the out of the assembly control rods and radial reflection of neutrons in the corner. The pin power distributions are highest for the most restrictive case and lowest for the least restrictive, as expected.

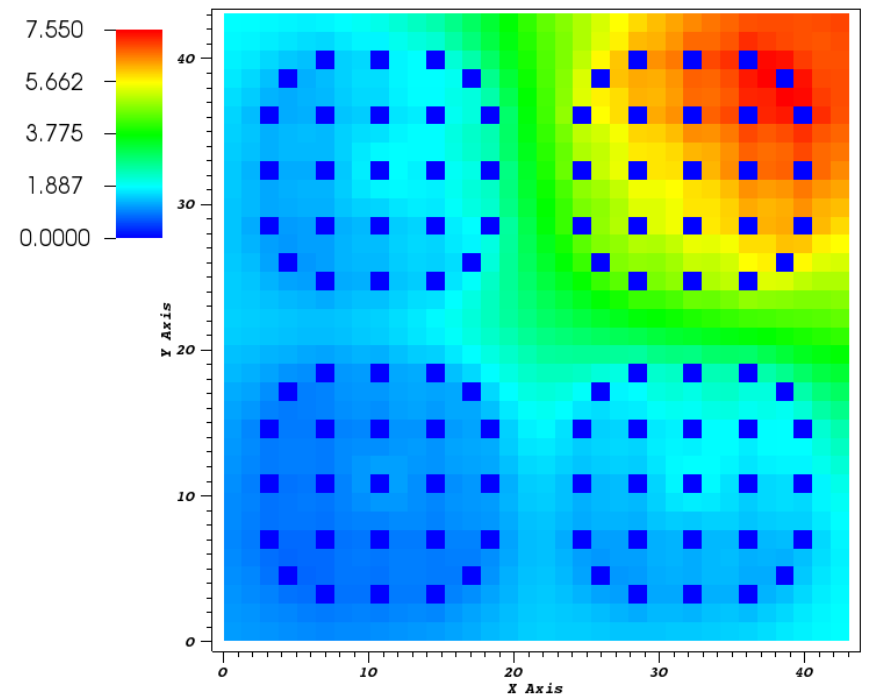

(a)

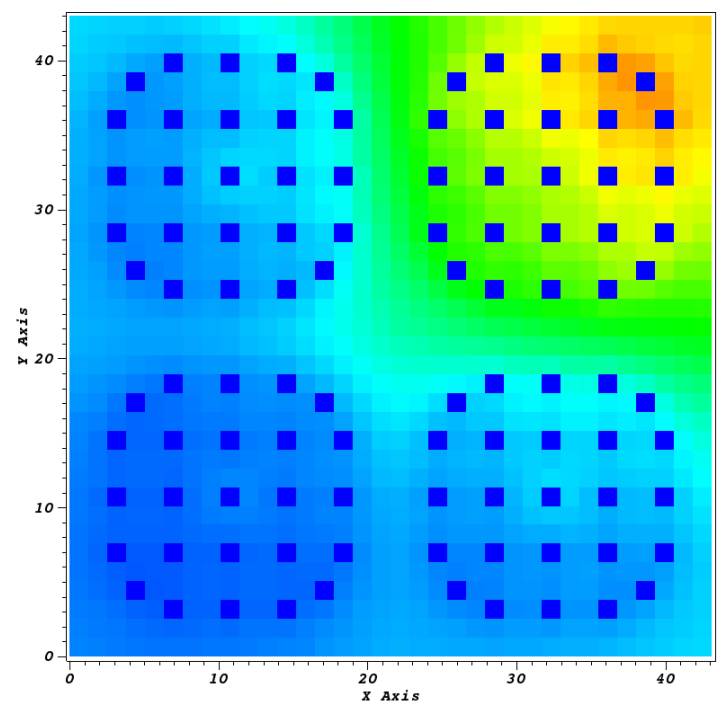

(c)

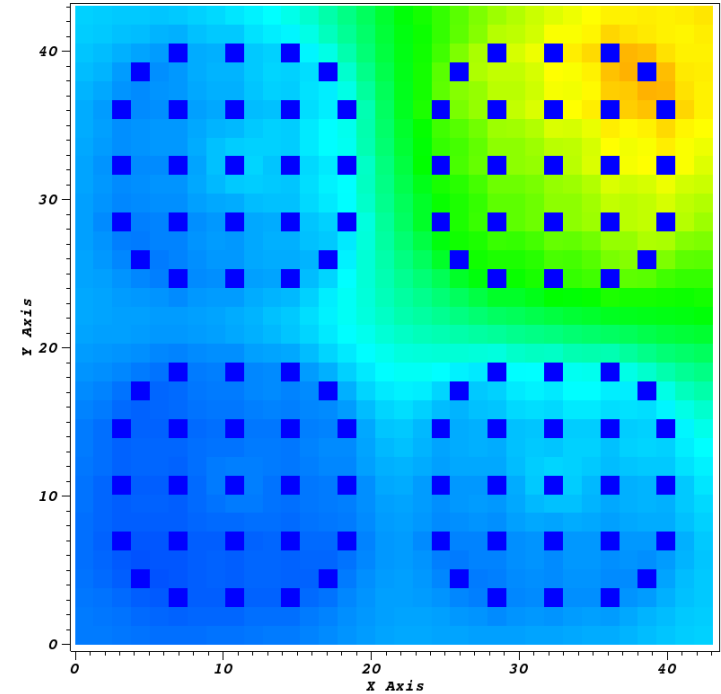

(b)
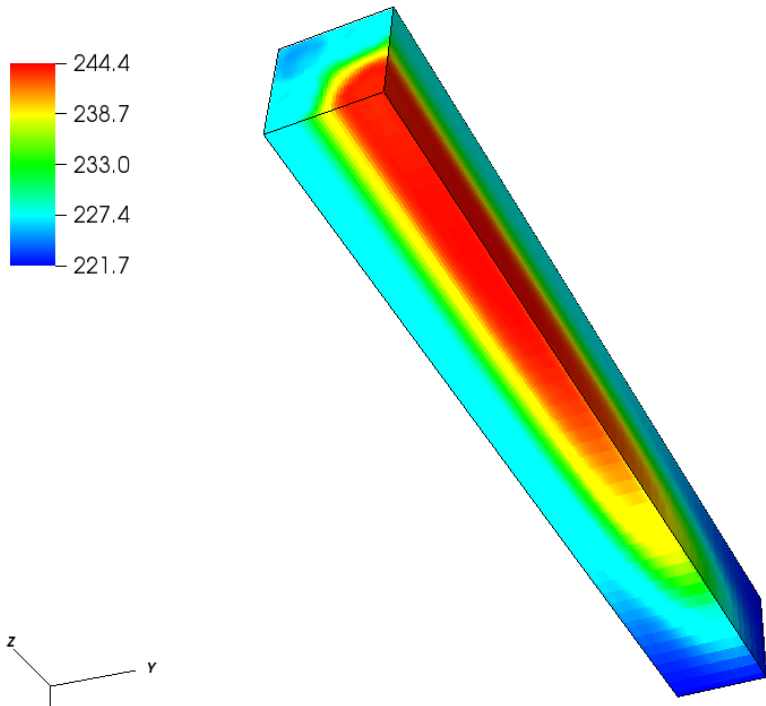

(d)

Figure 8. (a)-(c): Pin power distribution scaled by the legend in upper left for an axial slice of the 2x2 model at the location of MDNBR in the most restrictive (a), least restrictive (b), and nominal (c) cases. (d): The moderator temperature distribution in the $2 \times 2$ model for the most restrictive case. 


\subsection{Uncertainty Quantification Results}

Overall, 5143 VERA-CS 2x2 model simulations were performed for uncertainty quantification by use of the VUSAT. Table 5 shows the minimum, maximum, mean, and percentile values for each FOM. For the $95^{\text {th }}$ percentile, the standard error is shown calculated by the method in reference [17] to obtain the 95 percent probability at the 95 percent confidence level. In this case, the standard error at the $95^{\text {th }}$ percentile can be approximated as 2.11 multiplied by the standard error on the mean. Proving that a DNB event will not take place using the 95/95 criteria is the nuclear industry practice for reactor licensing. The current work therefore shows how this value will be obtained in uncertainty quantification using VERA-CS.

Table 5. FOM UQ values from the 5143 VERA-CS simulations.

\begin{tabular}{|c|c|c|c|c|c|c|}
\hline \multirow{2}{*}{ FOM } & \multirow{2}{*}{ Minimum } & \multicolumn{3}{|c|}{ Percentiles } & \multirow{2}{*}{ Maximum } & Mean \\
\cline { 3 - 6 } & & $5^{\text {th }}$ & $\mathbf{5 0}^{\text {th }}$ & $\begin{array}{c}\mathbf{9 5}^{\text {th }} \pm \text { Standard } \\
\text { Error }\end{array}$ & Mand \\
\hline MDNBR & 3.8015 & 5.0661 & 4.6794 & $\begin{array}{c}4.2000 \pm \\
7.7346 \times 10^{-3}\end{array}$ & 5.2502 & 4.6707 \\
\hline $\begin{array}{c}\text { Fuel Center-Line } \\
\left.\text { Temperature [ }{ }^{\circ} \mathrm{F}\right]\end{array}$ & 2042.35 & 2114.88 & 2263.38 & $\begin{array}{c}2501.88 \pm \\
3.44564\end{array}$ & 2736.68 & 2280.19 \\
\hline $\begin{array}{c}\text { Outer Clad Surface } \\
\left.\text { Temperature [ }{ }^{\circ} \mathrm{F}\right]\end{array}$ & 483.31 & 484.47 & 486.58 & $\begin{array}{c}489.46 \pm \\
4.3992 \times 10^{-2}\end{array}$ & 491.70 & 486.70 \\
\hline
\end{tabular}

Figure 9 shows the empirical PDF and one minus the empirical CDF for MDNBR. Figure 10 and Figure 11 show the empirical PDF and CDF for fuel center-line temperature and outer clad surface temperature, respectively. For each FOM, the PDF shape closely resembles a skewed Gaussian. From the near Gaussian shaped PDFs it is possible to deduce that the models that produce these distributions from the input distributions is quite simple [3]. Therefore, it is not expected that higher order methods of sensitivity analysis will be necessary (discussed further in section 4.3). In the case of MDNBR, one minus the empirical CDF is considered to obtain the percentile values in Table 5 rather than the actual CDF since the smaller MDNBR values are more limiting. This is opposite the maximum fuel center-line and outer clad surface temperatures where increased temperature values are more limiting and the CDF is used to obtain the percentile values in Table 5. 


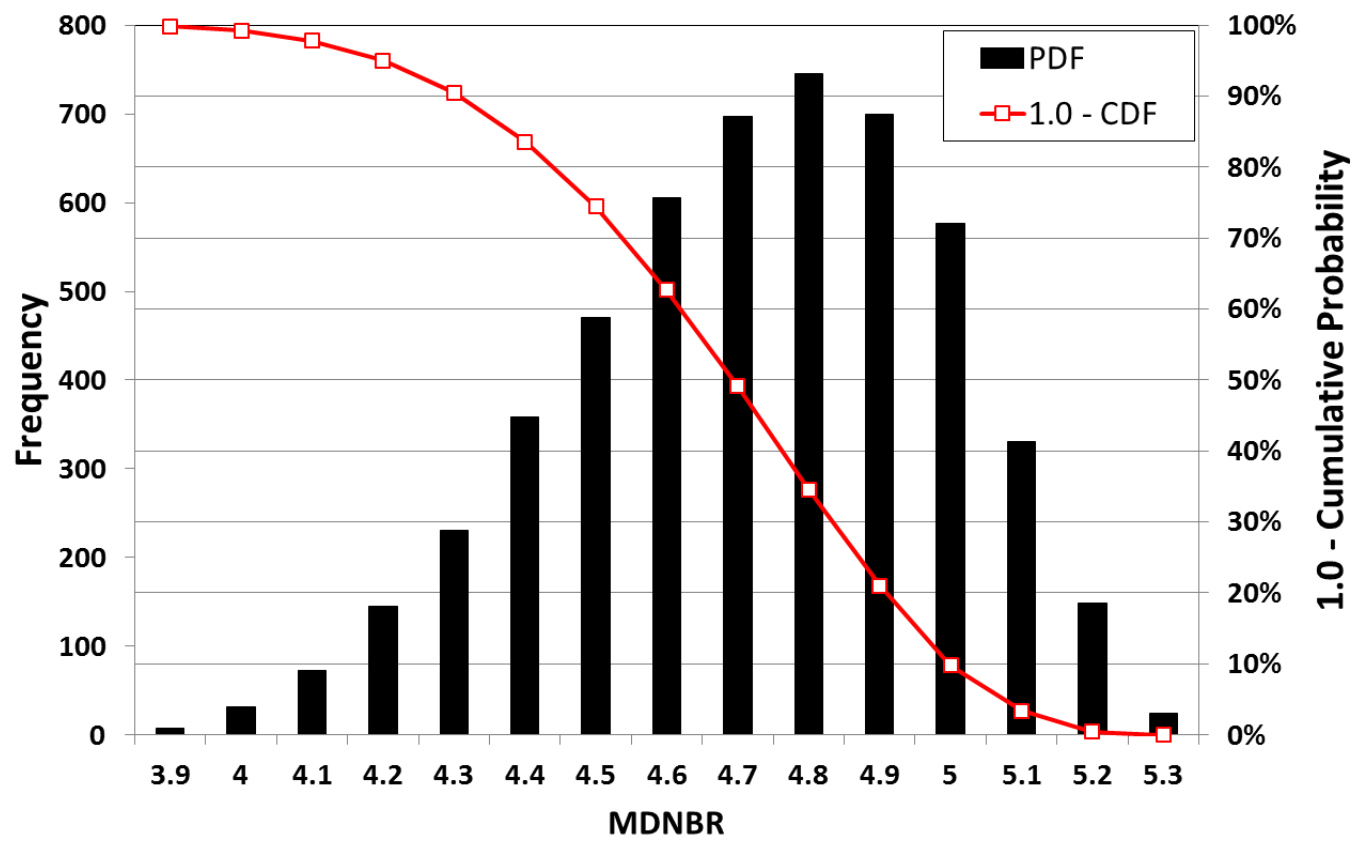

Figure 9. Empirical PDF and CDF for MDBNR.

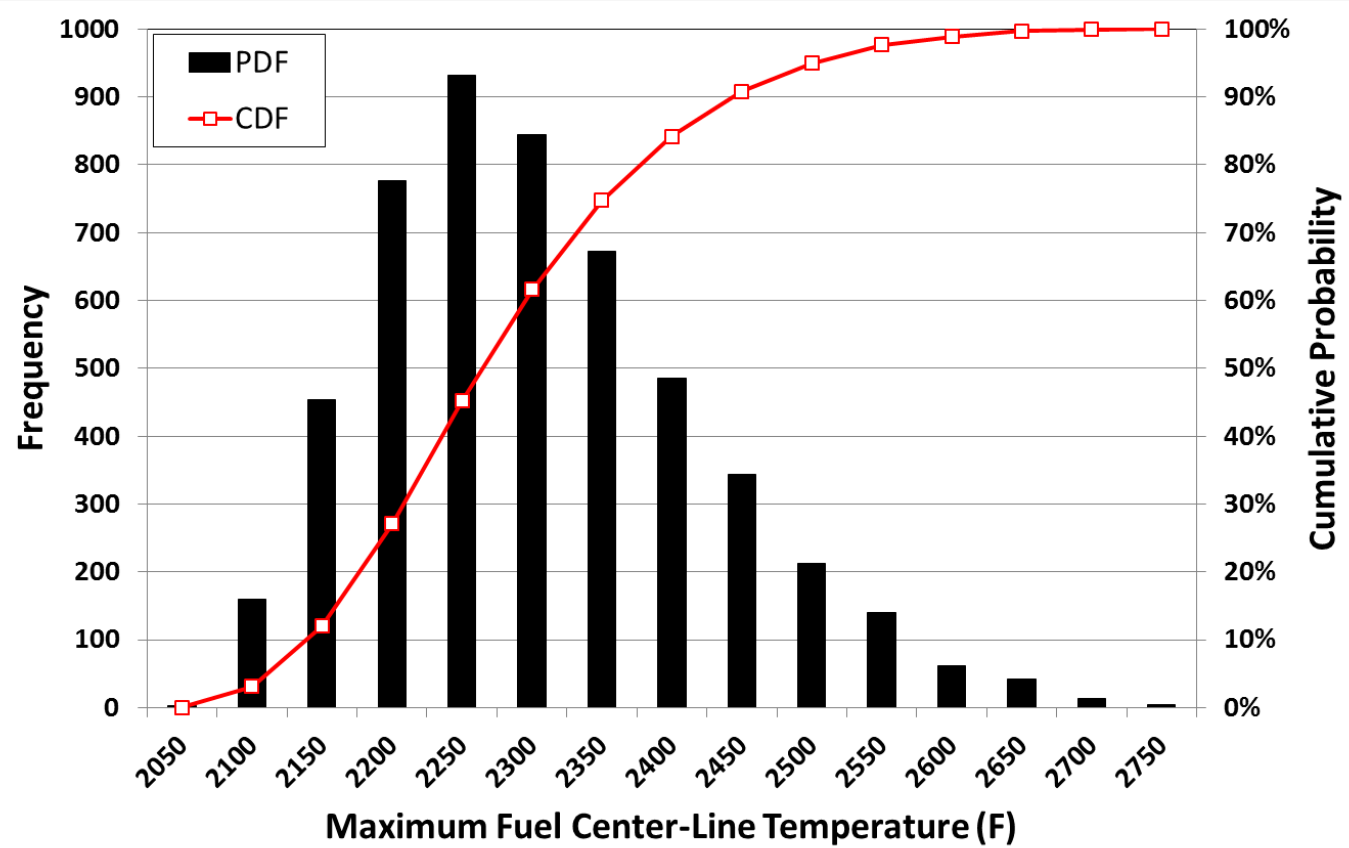

Figure 10. Empirical PDF and CDF for maximum fuel center-line temperature. 


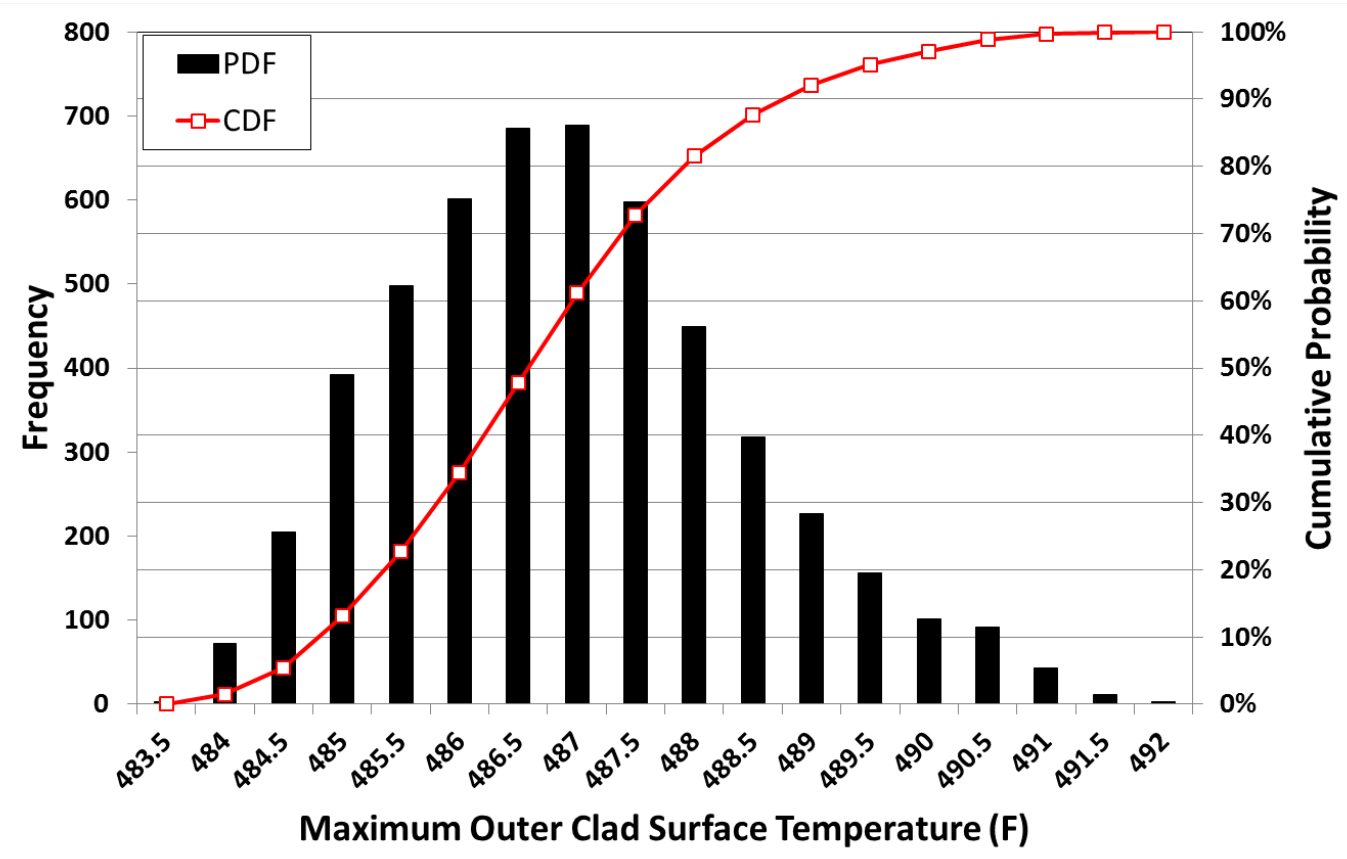

Figure 11. Empirical PDF and CDF for maximum outer clad surface temperature.

\subsection{Sensitivity Analysis Results}

The same data used for the uncertainty quantification study is examined for the sensitivity analysis of the 2x2 geometry with VERA-CS. The 3 FOMs and 14 input parameters were ranked by the method discussed in section 4.3 using the RANK function in Microsoft Excel to create scatterplots. The scatterplots for ranked MDNBR versus ranked input parameter are shown in Figure 12 and a trendline is used on each of the plots to show a linear fit to the data. Scatterplots for fuel center-line temperature and outer clad surface temperature were examined as well (Figure 13 and Figure 14). Those inputs that have a more prominent positive slope are the inputs that are most positively correlated with an increase in MDNBR. For example, the inlet mass flow rate has an obvious positive slope meaning that as the inlet mass flow rate is increased, the MDNBR increases as more heat is removed by the coolant. Conversely, those inputs that have a prominent negative slope indicate a negative correlation between the input and MDNBR. For example, as the inlet temperature is increased the critical heat flux condition is exacerbated, decreasing the MDNBR. Notably, some cases do not have a particularly strong correlation with MDNBR. However, correlation coefficients are needed to quantify the contributions of the uncertainty in each input parameter to changes in each FOM.

The Pearson correlation coefficients between each of the perturbed parameters and considered FOMs were calculated using the PEARSON function in Microsoft Excel. The Spearman coefficients were also calculated by use of the CORREL function in Microsoft Excel on the rank transformed data and results were consistent with the Pearson correlation coefficients. The sum of the $R^{2}$ values were examined for each FOM to ensure that Pearson correlation coefficients were suitable for analyzing model correlations and for each of the FOMs the $R^{2}$ values was equal to, or nearly equal to, unity. However, partial correlation coefficients are shown here as well to demonstrate the application of sensitivity analysis techniques to VERA-CS. PCCs were calculated using an Excel VBA script based on that available publicly by Listen Data [18]. Values for all of the correlation coefficients for each FOM are shown in Table 6 - Table 8 along with the importance rank of each particular parameter. The results show that the inlet temperature is the most important parameter across each of the FOMs, regardless of the correlation coefficient calculation technique. 
The most correlated parameters are consistent for each of the correlation techniques for at least the first four most influential parameters after which there are some differences. Interestingly, some sign change is noticed between correlation coefficient techniques for the least correlated values (e.g. fuel enrichment for MDNBR). In general, the largest differences in importance occur for the spacer grid loss coefficients between the CC/RCC values and the PCC values. However, PCCs can give misleading results when correlations exist between model inputs [1] and this effect may be shown here between the correlated spacer grid form loss inputs.

Table 6. Summary of correlation coefficients and importance rank for MDBNR.

\begin{tabular}{|c|c|c|c|c|c|c|}
\hline \multicolumn{7}{|c|}{ MDNR } \\
\hline Parameter & CC & CC Rank & RCC & $\begin{array}{c}\text { RCC } \\
\text { Rank }\end{array}$ & PCC & PCC Rank \\
\hline Power & -0.4324 & 3 & -0.4259 & 3 & -0.9620 & 3 \\
\hline Inlet Temperature & -0.6141 & 1 & -0.6134 & 1 & -0.9806 & 1 \\
\hline System Pressure & 0.3149 & 4 & 0.3076 & 4 & 0.9329 & 4 \\
\hline $\begin{array}{c}\text { Inlet Mass Flow } \\
\text { Rate }\end{array}$ & 0.5482 & 2 & 0.5436 & 2 & 0.9750 & 2 \\
\hline$K_{m}$ & -0.0102 & 12 & -0.0080 & 13 & -0.0026 & 14 \\
\hline$\beta_{\text {sp }}$ & 0.0207 & 9 & 0.0196 & 10 & 0.0378 & 9 \\
\hline dhfrac & 0.0184 & 11 & 0.0171 & 11 & 0.1683 & 8 \\
\hline $\begin{array}{c}\text { Spacer Grid Form } \\
\text { Loss - MID }\end{array}$ & 0.0458 & 6 & 0.0425 & 6 & -0.0093 & 13 \\
\hline $\begin{array}{c}\text { Spacer Grid Form } \\
\text { Loss - END }\end{array}$ & 0.0458 & 7 & 0.0425 & 7 & 0.0093 & 12 \\
\hline Fuel Pellet Radius & -0.0053 & 13 & -0.0058 & 14 & -0.0093 & 11 \\
\hline Clad Inner Radius & 0.0296 & 8 & 0.0305 & 8 & 0.2614 & 6 \\
\hline Clad Outer Radius & 0.1767 & 5 & 0.1713 & 5 & 0.8035 & 5 \\
\hline $\begin{array}{c}\text { Fuel \% of } \\
\text { Theoretical Density }\end{array}$ & -0.0195 & 10 & -0.0202 & 9 & -0.2316 & 7 \\
\hline Fuel Enrichment & -0.0025 & 14 & -0.0082 & 12 & 0.0100 & 10 \\
\hline
\end{tabular}




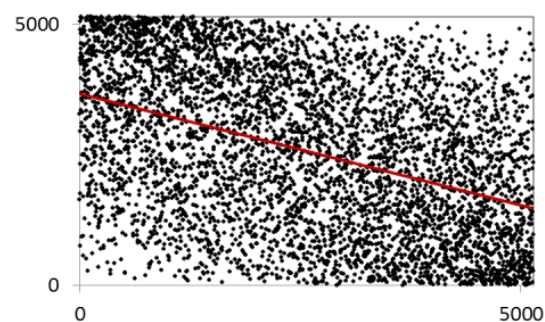

(a) Power

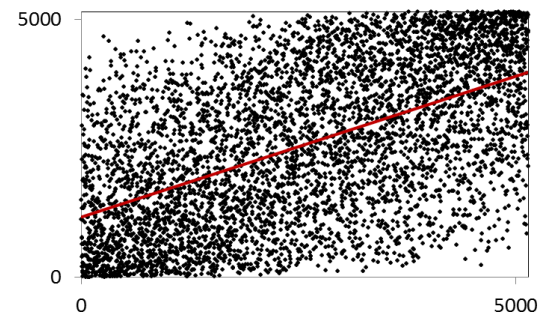

(d) Inlet Mass Flow

Rate

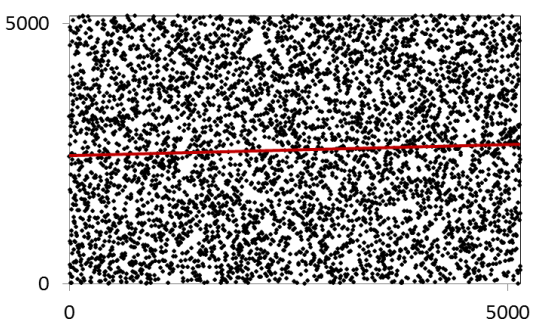

(g) Spacer Grid Form

Loss - MID
5000

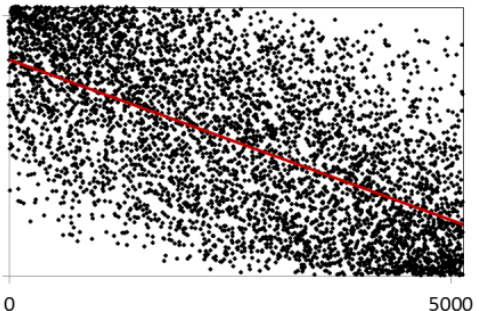

(b) Inlet Temperature

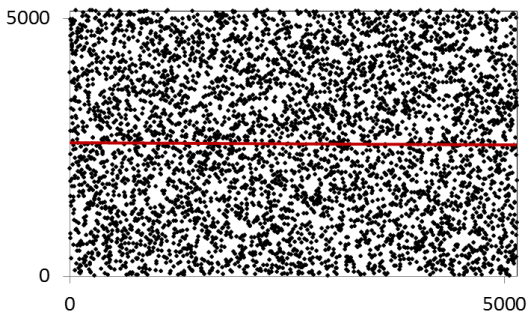

(e) $K_{m}$

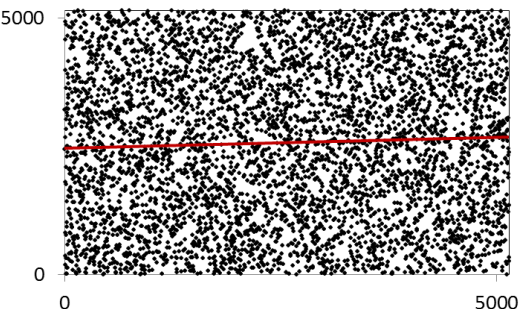

(h) Spacer Grid Form Loss - END

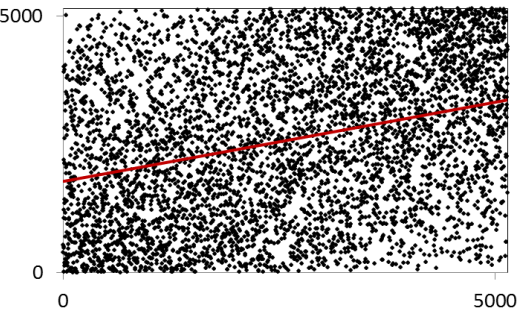

(c) System Pressure

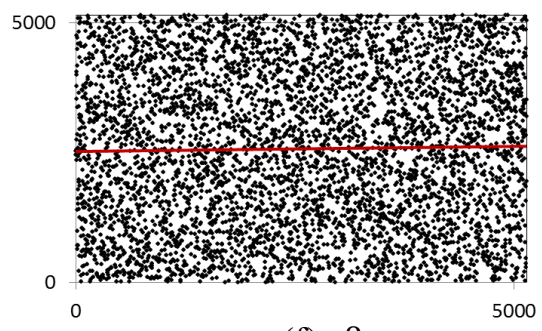

(f) $\beta_{\mathrm{sp}}$

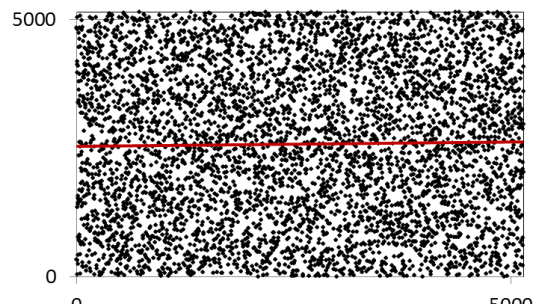

(i) dhfrac

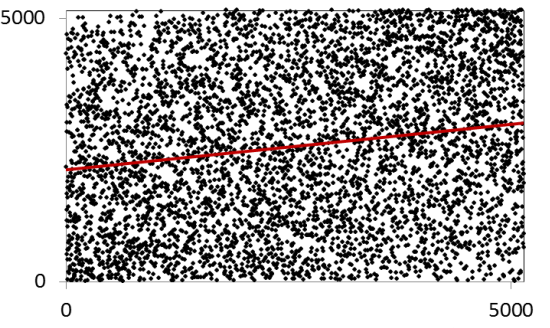

(l) Clad Outer Radius

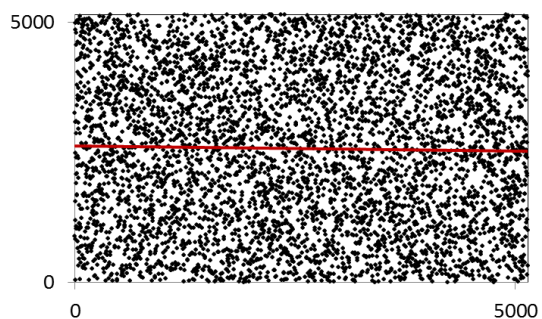

(m) Fuel \% of Theoretical Density

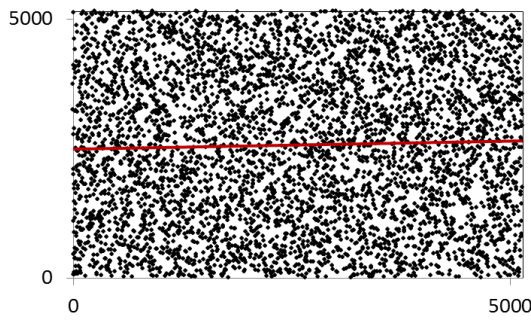

(k) Clad Inner Radius

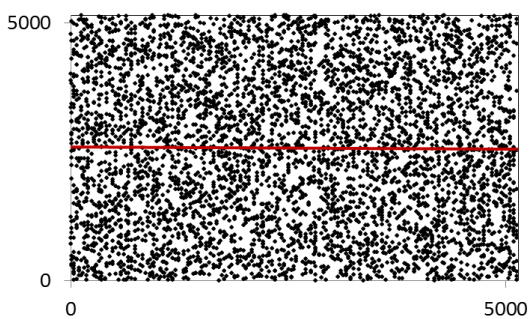

(n) Fuel Enrichment

Figure 12. Ranked MDNBR versus ranked input parameter for sensitivity analysis. 


\subsubsection{Pearson correlation coefficients}

As mentioned previously, the sum of the $R^{2}$ values indicate that CCs are adequate for sensitivity analysis pertaining to the considered VERA-CS model and a discussion specific to the CC results will be provided here. For MDNBR (Table 6) the inlet temperature, inlet mass flow rate, power, and system pressure play the most important roles, respectively. This importance ranking of the perturbed parameters is not unexpected since the critical heat flux and DNBR calculations are a function of these inputs. The next four importance parameters (clad outer radius, spacer grid form loss coefficients, and clad inner radius) can all be physically linked to the MDNBR calculation as well although the amount of correlation decreases significantly beyond the clad outer radius. As clad outer radius changes so does the overall coolant flow area and therefore the coolant mass flux. The spacer grid form loss coefficients contribute to changes in mass flow rate since larger coefficient values create a larger loss term in the CTF momentum equations. The combination of increasing or decreasing the clad inner and outer radii changes the clad thickness and therefore the amount of heat that is dissipated to the clad itself. The remaining terms are essentially insignificant to MDNBR. This is an interesting result since the void-drift coefficient $\left(K_{m}\right)$, turbulent mixing coefficient $\left(\beta_{\mathrm{sp}}\right)$, and gamma heating (dhfrac) all have large input uncertainty. Also, the fuel pellet radius, theoretical density, and enrichment would intuitively seem to affect the heat generation and transfer realized by the MDNBR. However, sensitivity analysis has shown that this is not the case. All of the trends in CC values for MDNBR are accurately represented by the scatterplots in Figure 12.

Sensitivity analysis based on CC results for the fuel center-line temperature (Table 7) show that inlet temperature, power, inlet mass flow rate, and system pressure are the most important parameters, respectively. These parameters are correlated oppositely from MDNBR since the FOMs themselves are oppositely desired, i.e. higher MDNBR and lower fuel center-line temperatures are desired from a reactor safety perspective. The next important parameter is the fuel density, which affects the heat transfer directly out of the fuel pellet. The remaining parameters are ranked much like that of MDNBR, with the exception of clad outer radius being much less important, and are essentially insignificant. Again, from a heat transfer standpoint it is somewhat counterintuitive that fuel pellet radius is unimportant but results from sensitivity analysis show this to be the case. Larger uncertainty in fuel pellet radius could increase the importance but would be non-physical based on fresh fuel pellet manufacturing tolerance. Later in fuel cycle life, when fuel swelling becomes substantial, the fuel pellet radius could become more of a contributing factor in the selected FOMs. All of the trends in CC values for the fuel center-line temperature are represented in the scatterplots in Figure 13. 
Table 7. Summary of correlation coefficients and importance rank for fuel center-line temperature.

\begin{tabular}{|c|c|c|c|c|c|c|}
\hline \multicolumn{7}{|c|}{ Fuel Center-Line Temperature } \\
\hline Parameter & CC & CC Rank & RCC & $\begin{array}{c}\text { RCC } \\
\text { Rank }\end{array}$ & PCC & PCC Rank \\
\hline Power & 0.5001 & 2 & 0.5088 & 2 & 0.9218 & 2 \\
\hline Inlet Temperature & 0.5846 & 1 & 0.5831 & 1 & 0.9424 & 1 \\
\hline System Pressure & -0.3034 & 4 & -0.2994 & 4 & -0.8231 & 4 \\
\hline $\begin{array}{c}\text { Inlet Mass Flow } \\
\text { Rate }\end{array}$ & -0.4483 & 3 & -0.4422 & 3 & -0.9040 & 3 \\
\hline$K_{m}$ & 0.0172 & 12 & 0.0166 & 12 & 0.0035 & 14 \\
\hline$\beta_{\text {sp }}$ & -0.0252 & 9 & -0.0290 & 9 & -0.0429 & 8 \\
\hline dhfrac & -0.0187 & 11 & -0.0188 & 10 & -0.1254 & 7 \\
\hline $\begin{array}{c}\text { Spacer Grid Form } \\
\text { Loss - MID }\end{array}$ & -0.0550 & 7 & -0.0488 & 7 & 0.0107 & 13 \\
\hline $\begin{array}{c}\text { Spacer Grid Form } \\
\text { Loss - END }\end{array}$ & -0.0550 & 8 & -0.0488 & 8 & -0.0107 & 12 \\
\hline Fuel Pellet Radius & 0.0053 & 13 & 0.0087 & 13 & -0.0203 & 10 \\
\hline Clad Inner Radius & -0.0585 & 6 & -0.0562 & 6 & -0.2792 & 6 \\
\hline Clad Outer Radius & -0.0211 & 10 & -0.0167 & 11 & -0.0376 & 9 \\
\hline $\begin{array}{c}\text { Fuel \% of } \\
\text { Theoretical Density }\end{array}$ & -0.2796 & 5 & -0.2875 & 5 & -0.7944 & 5 \\
\hline Fuel Enrichment & -0.0012 & 14 & 0.0054 & 14 & -0.0186 & 11 \\
\hline
\end{tabular}

The sensitivity analysis results based on CC for outer clad surface temperature (Table 8) are considerably different than those of MDNBR or fuel center-line temperature. The coolant inlet temperature is again the most important parameter but possesses a much larger portion of the variance, with a CC value of over 0.73 for the outer clad surface temperature in comparison to about 0.61 for the MDNBR and roughly 0.58 for the fuel center-line temperature. The inlet mass flow rate and power follow as the next important parameters. These first three input parameters are directly related to how much heat is removed from the outer surface of the fuel cladding. The next important inputs, clad outer radius and clad inner radius, have much smaller CC values than equally ranked parameters for MDNBR and fuel center-line temperature although they would both influence clad heat transfer characteristics. Interestingly, the turbulent mixing coefficient $\left(\beta_{\mathrm{sp}}\right)$ does have a higher ranked importance in terms of outer clad surface temperature. The remaining parameters are ranked much as the other FOMs while contributing much less to the overall variance in outer clad surface temperature. The trends in CC values for the outer clad surface temperature are represented in the scatterplots in Figure 14. 
Table 8. Summary of correlation coefficients and importance rank for outer clad surface temperature.

\begin{tabular}{|c|c|c|c|c|c|c|}
\hline \multicolumn{7}{|c|}{ Outer Clad Surface Temperature } \\
\hline Parameter & CC & CC Rank & RCC & $\begin{array}{c}\text { RCC } \\
\text { Rank }\end{array}$ & PCC & PCC Rank \\
\hline Power & 0.3893 & 3 & 0.3764 & 3 & 0.9279 & 3 \\
\hline Inlet Temperature & 0.7342 & 1 & 0.7453 & 1 & 0.9779 & 1 \\
\hline System Pressure & -0.0072 & 13 & 0.0058 & 14 & -0.0578 & 8 \\
\hline $\begin{array}{c}\text { Inlet Mass Flow } \\
\text { Rate }\end{array}$ & -0.5373 & 2 & -0.5263 & 2 & -0.9582 & 2 \\
\hline$K_{m}$ & 0.0128 & 10 & 0.0086 & 12 & 0.0091 & 11 \\
\hline$\beta_{\text {sp }}$ & -0.0241 & 6 & -0.0220 & 8 & -0.0566 & 9 \\
\hline dhfrac & -0.0086 & 11 & -0.0095 & 10 & -0.0869 & 7 \\
\hline $\begin{array}{c}\text { Spacer Grid Form } \\
\text { Loss - MID }\end{array}$ & 0.0217 & 8 & 0.0255 & 7 & 0.0086 & 13 \\
\hline $\begin{array}{c}\text { Spacer Grid Form } \\
\text { Loss - END }\end{array}$ & 0.0217 & 7 & 0.0255 & 6 & -0.0086 & 14 \\
\hline Fuel Pellet Radius & 0.0080 & 12 & 0.0095 & 11 & 0.0088 & 12 \\
\hline Clad Inner Radius & -0.0269 & 5 & -0.0282 & 5 & -0.1913 & 5 \\
\hline Clad Outer Radius & -0.0850 & 4 & -0.0824 & 4 & -0.4215 & 4 \\
\hline $\begin{array}{c}\text { Fuel \% of } \\
\text { Theoretical Density }\end{array}$ & 0.0195 & 9 & 0.0211 & 9 & 0.1514 & 6 \\
\hline Fuel Enrichment & 0.0018 & 14 & 0.0065 & 13 & -0.0232 & 10 \\
\hline
\end{tabular}

\section{CONCLUSIONS}

This study was performed to develop an uncertainty quantification and sensitivity analysis approach to the CASL nuclear reactor core simulation code VERA-CS. VERA-CS is a fully coupled code for core neutronics and thermal-hydraulic calculations and eliminates the uncertainty in core power factor inputs seen in past UQ/SA studies in nuclear thermal hydraulic applications. The VUSAT was developed to handle the UQ/SA approach with the ability to perturb any number of selected input parameters, create an arbitrary number of perturbed VERA-CS input files, and post-process the VERA-CS cases to create a single output file containing the FOM and perturbed parameter values for each case. A table of uncertain parameters was developed for the 2x2 geometry considered in this study with inputs expected to influence the FOMs. The results of sensitivity analysis show that a number of correlation coefficients can be calculated for the Monte Carlo global sensitivity analysis considered in this work. Sensitivity analysis results also show that those parameters used as inputs to the $\mathrm{W}-3$ boiling correlation are most influential while highly modeled parameters $\left(K_{m}, \beta_{\mathrm{sp}}\right)$ are less influential.

Future work for UQ and SA with VERA-CS will focus on improving the 2x2 geometry set-up to more closely mirror the area of interest in a full-core MSLB scenario. Also, Monte Carlo UQ/SA of full-core geometries is on the horizon with the continually increasing capabilities of high performance computing.

\section{ACKNOWLEDGEMENT}

This research made use of the resources of the High Performance Computing Center at Idaho National Laboratory, which is supported by the Office of Nuclear Energy of the U.S. Department of Energy under Contract No. DE-AC07-05ID14517. The authors would like to thank Andrew Godfrey and Mark Baird at ORNL as well as Vefa Kucukboyaci and Yixing Sung at Westinghouse for their assistance with VERACS. This manuscript has been authored by Battelle Energy Alliance, LLC under Contract No. DE-AC0705ID14517 with the U.S. Department of Energy. The United States Government retains and the publisher, 
by accepting the article for publication, acknowledges that the United States Government retains a nonexclusive, paid-up, irrevocable, world-wide license to publish or reproduce the published form of this manuscript, or allow others to do so, for United States Government purposes. 


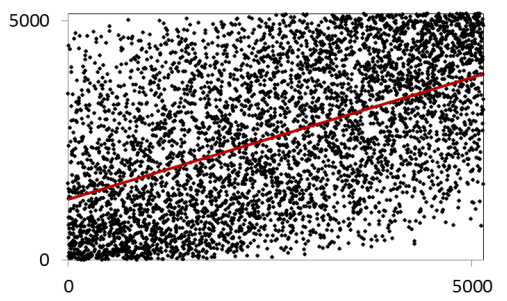

(a) Power

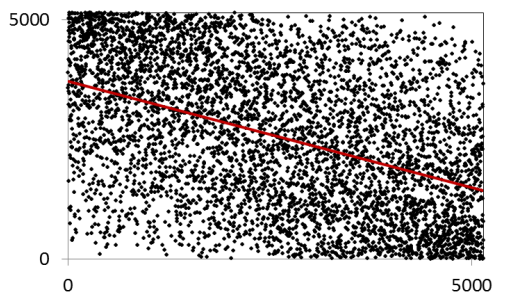

(d) Inlet Mass Flow Rate

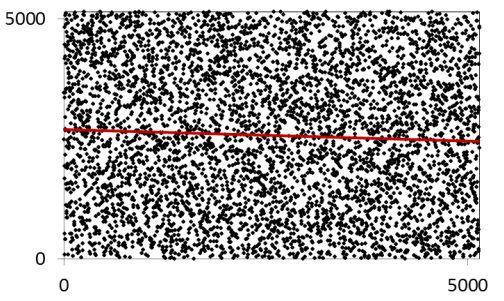

(g) Spacer Grid Form Loss - MID

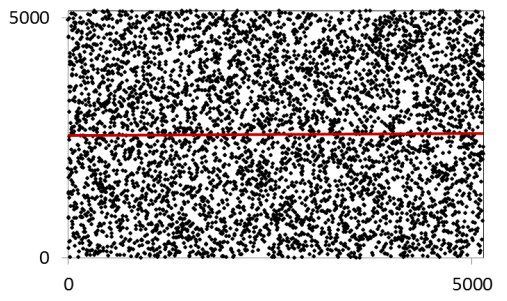

(j) Fuel Pellet Radius

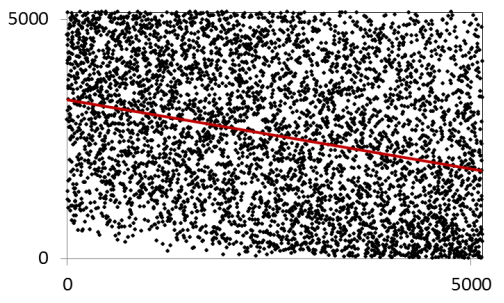

(m) Fuel \% of Theoretical Density

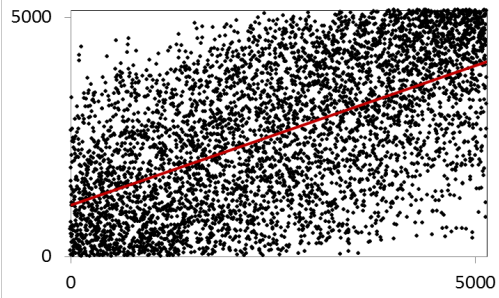

(b) Inlet Temperature

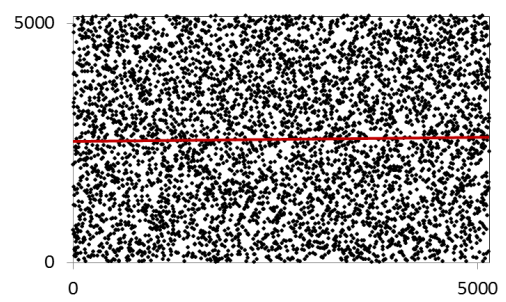

(e) $K_{m}$

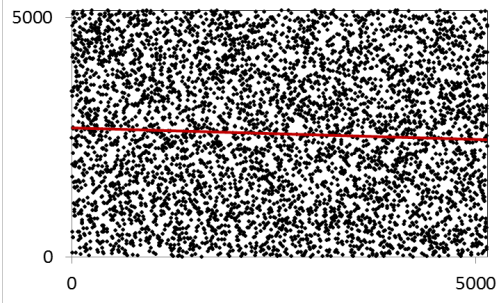

(h) Spacer Grid Form Loss - END

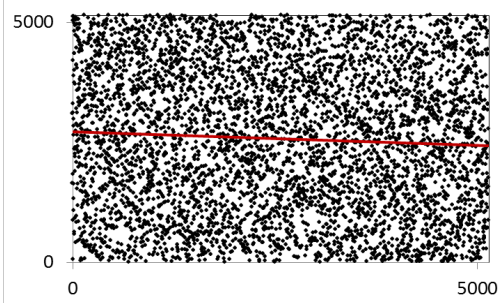

(k) Clad Inner Radius

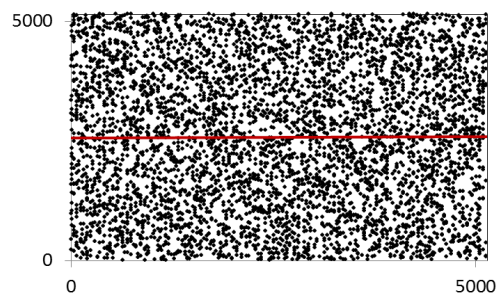

(n) Fuel Enrichment

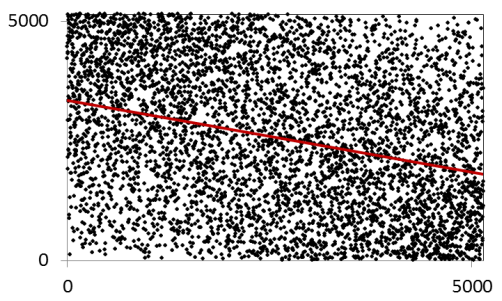

(c) System Pressure

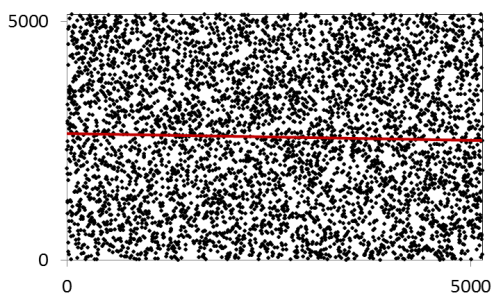

(f) $\beta_{\mathrm{sp}}$

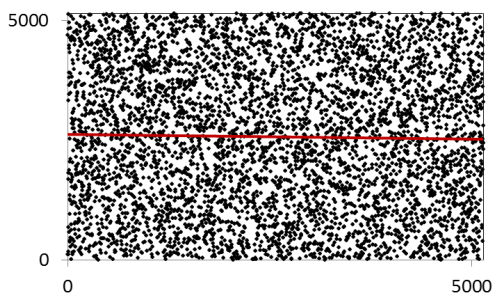

(i) dhfrac

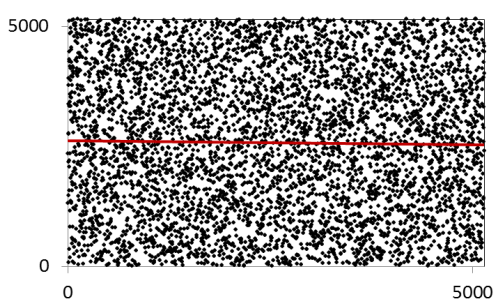

(l) Clad Outer Radius

Figure 13. Ranked fuel center-line temperature versus ranked input parameter for sensitivity analysis. 


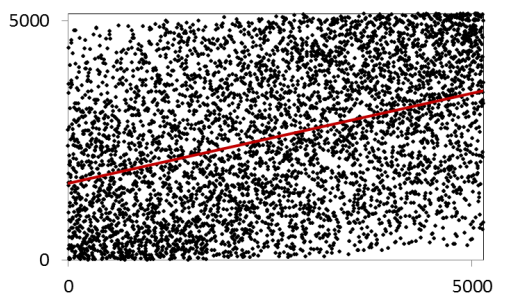

(a) Power

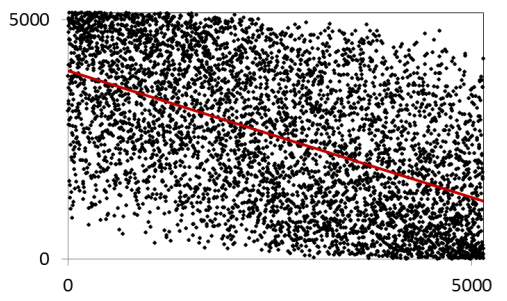

(d) Inlet Mass Flow Rate

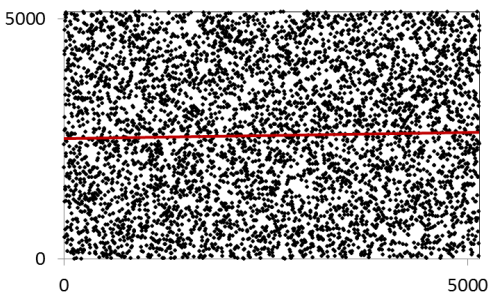

(g) Spacer Grid Form Loss - MID

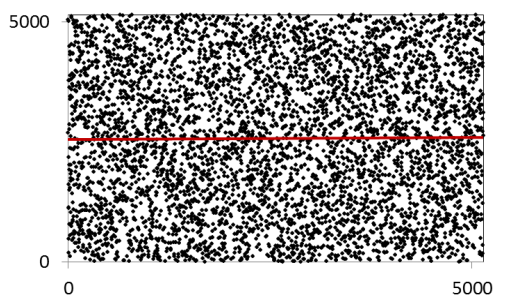

(j) Fuel Pellet Radius

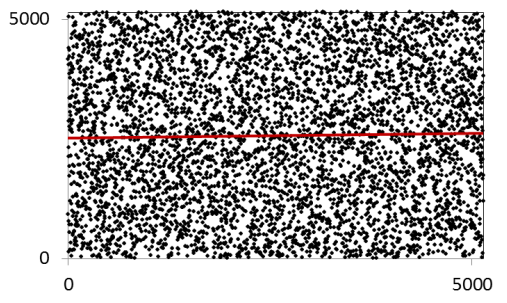

(m) Fuel \% of Theoretical Density

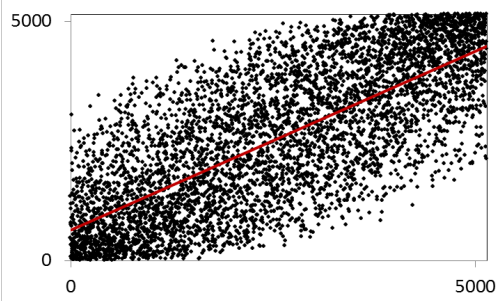

(b) Inlet Temperature

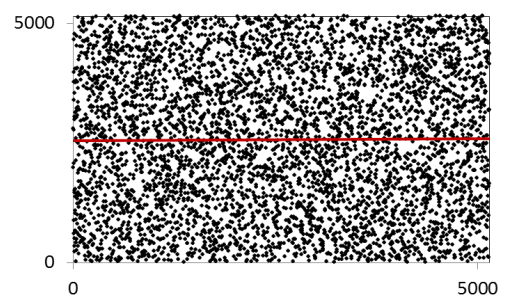

(e) $K_{m}$

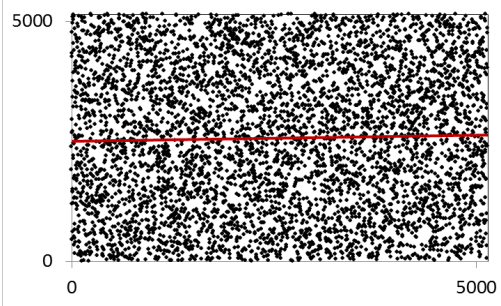

(h) Spacer Grid Form Loss - END

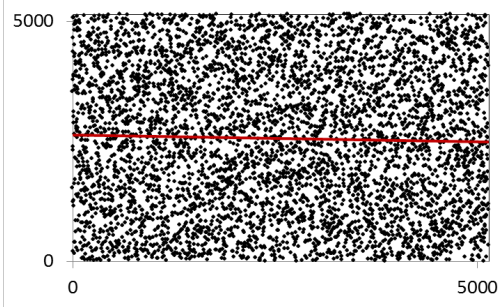

(k) Clad Inner Radius

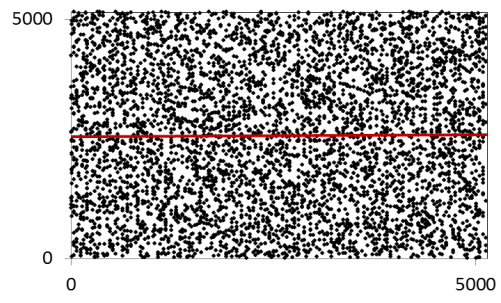

(n) Fuel Enrichment

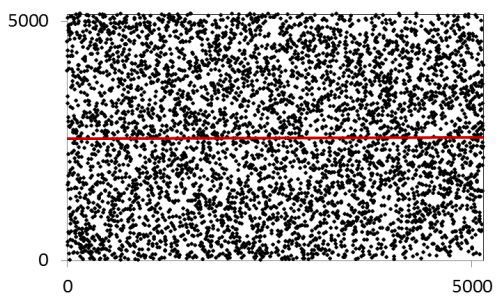

(c) System Pressure

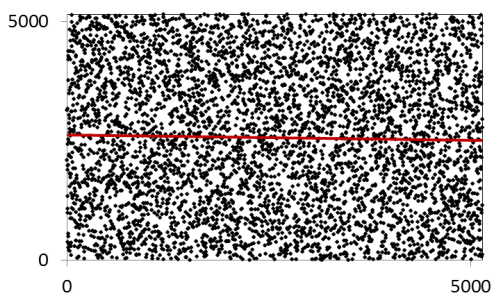

(f) $\beta_{\mathrm{sp}}$

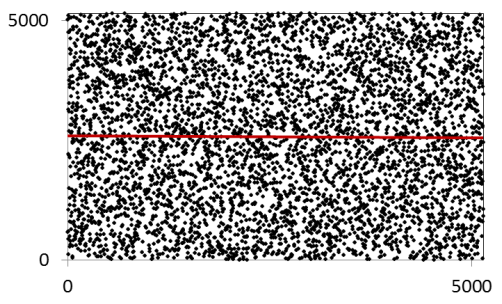

(i) dhfrac

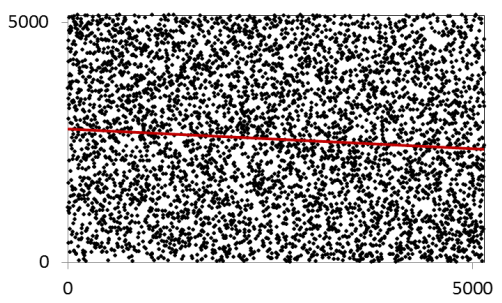

(l) Clad Outer Radius

Figure 14. Ranked outer clad surface temperature versus ranked input parameter for sensitivity analysis. 


\section{REFERENCES}

[1] J. C. Helton, J. D. Johnson, C. J. Sallaberry and C. B. Storlie, "Survey of sampling-based methods for uncertainty and sensitivity analysis," SANDIA Report SAND2006-2901, June 2006.

[2] W. R. Marcum and A. J. Brigantic, "Applying uncertainty and sensitivity on thermal hydraulic subchannel analysis for the multi-application small light water reactor," Nuclear Engineering and Design, Vol. 293, pp. 272-291, 2015.

[3] T. Ikonen, "Comparison of global sensitivity analysis methods - Application to fuel behavior modeling," Nuclear Engineering and Design, Vol. 297, pp. 72-80, 2016.

[4] B. Collins and A. Godfrey, "Analysis of the BEAVRS benchmark using VERA-CS," in ANS MC2015 - Joint International Conference on Mathematics and Computation (M\&C), Supercomputing in Nuclear Applications (SNA), and the Monte Carlo (MC) Method, Nashville, TN, April 19-23, 2015.

[5] F. Franceshini, A. T. Godfrey, S. Stimpson, T. Evans, B. Collins, J. C. Gehin, J. Turner, A. Graham and T. Downar, "AP1000 PWR Startup core modeling and simulation with VERA-CS," in Advances in Nuclear Fuel Management V (ANFM 2015) , Hilton Head Island, South Carolina, USA, March 29 - April 1, 2015.

[6] B. Kochunas, B. Collins, D. Jabaay, S. Stimpson, A. Graham, K. S. Kim, W. Wieselquist, K. Clarno, S. Palmtag, T. Downar and J. Gehin, "VERA core simulator methodology for PWR cycle depletion," in ANS MC2015 - Joint International Conference on Mathematics and Computation (M\&C), Supercomputing in Nuclear Applications (SNA), and the Monte Carlo (MC) Method, Nashville, TN, April 19-23, 2015.

[7] A. Godfrey , B. Collins, K. S. Kim, R. Lee, J. Powers, R. Salko, S. Stimpson, W. Wieselquist, R. Montgomery, R. Montgomery, B. Kochunas, D. Jabaay, N. Capps and J. Secker, "VERA benchmarking results for Watts Bar Nuclear Plant Unit 1 cycles 1-12," CASL Report CASL-U-20150206-000, June 30, 2015.

[8] B. Collins, S. Stimpson, B. Kochunas, T. Downar and W. Martin, "Assessment of 2D/1D capability in MPACT," in PHYSOR 2014, Kyoto, Japan, Sept. 28 - Oct. 3, 2014.

[9] R. K. Salko, T. Lange, V. Kucukboyaci, Y. Sung, S. Palmtag, J. Gehin and M. Avramova, "Development of COBRA-TF for modeling full-core reactor operating cycles," in Advances in Nuclear Fuel Management V (ANFM 2015) , Hilton Head Island, SC, USA, March 29 - April 1, 2015.

[10] I. C. Gauld, G. Radulescu, G. Ilas, B. D. Murphy, M. L. Williams and D. Wiarda, "Isotopic depletion and decay methods and analysis capabilities in SCALE," Nuclear Technology, Vol. 174, no. 2, p. 169, 2011.

[11] Y. Sung, V. Kucukboyaci, Y. Xu and R. K. Salko, "VERA-CS modeling and simulation of PWR MSLB core response to DNB - high flow case," CASL Report CASL-X-2015-0174-000, June 30, 2015.

[12] S. Palmtag and A. Godfrey, "VERA Common Input User Manual," CASL CASL-U-2014-0014-002, February 23, 2015.

[13] L. S. Tong, "Prediction of departure from nucleate boiling for an axially non-uniform heat flux distribution," Journal of Nuclear Energy, Vol. 21, pp. 241-248, 1967.

[14] J. M. Doster, "Assessment of the performance of COBRA-TF for the prediction of subcooled boiling conditions in rod bundles," CASL Report CASL-U-2013-0201-000, 2013.

[15] C. Frepoli, "An overview of Westinghouse realistic large break LOCA Evaluation Model," Science and Technology of Nuclear Installations, Vol. 2008, Article ID 498737, 2008.

[16] R. P. Martin and L. D. O'Dell, "AREVA's realistic large break LOCA analysis methodology," 
Nuclear Engineering and Design, Vol. 235, pp. 1713-1725, 2005.

[17] B. Harding, C. Tremblay and D. Cousineau, "Standard errors: A review and evaluation of standard error estimators using Monte Carlo simulations," The Quantitative Methods For Psychology, Vol. 10, no. 2, 2014.

[18] "Calculating partial correlation coefficients with Excel VBA," Listen Data, 2015. [Online]. Available: http://www.listendata.com/2013/02/excel-udf-partial-correlation-matrix.html. 